JOURNAL OF THE

AMERICAN MATHEMATICAL SOCIETY

Volume 25, Number 3, July 2012, Pages 667-713

S 0894-0347(2012)00735-8

Article electronically published on March 26, 2012

\title{
MICROSCOPIC DERIVATION OF GINZBURG-LANDAU THEORY
}

\author{
RUPERT L. FRANK, CHRISTIAN HAINZL, ROBERT SEIRINGER, \\ AND JAN PHILIP SOLOVEJ
}

\section{Contents}

1. Introduction and main results

2. Semiclassical estimates

3. Preliminaries

4. Proof of Theorem 11 Upper bound

5. Proof of Theorem 11 Lower bound, Part A

6. Proof of Theorem 1 Lower bound, Part B

7. Proof of Theorem 2

8. Proof of Theorem 3

Appendix A. Properties of $\alpha_{0}$

References

\section{INTRODUCTION AND MAIN RESUltS}

1.1. Introduction. In 1950, Ginzburg and Landau [7] introduced a model of superconductivity that has been extremely successful and is widely used in physics, even beyond the theory of superconductivity. It has a rich mathematical structure that has been studied in great detail and has inspired the development of many interesting new concepts. Ginzburg and Landau arrived at their model in a phenomenological way, describing the macroscopic properties of a superconductor, without the need to understand the microscopic mechanism.

In 1957, Bardeen, Cooper and Schrieffer [2] formulated the first microscopic explanation of superconductivity starting from a many-body Hamiltonian. In a major breakthrough they realized that this phenomenon can be described by a pairing mechanism. Below a critical temperature, a superconducting paired state

Received by the editors February 20, 2011 and, in revised form, November 14, 2011.

2010 Mathematics Subject Classification. Primary 35A15, 81Q20, 82D50, 82D55, 35Q56.

The first author gratefully acknowledges financial support received via U.S. NSF grant PHY1068285 .

The second author gratefully acknowledges financial support received via U.S. NSF grant DMS0800906.

The third author gratefully acknowledges financial support received via U.S. NSF grant PHY0845292 and NSERC.

The last author gratefully acknowledges financial support received via a grant from the Danish council for independent research. 
forms due to an instability of the normal state in the presence of an attraction between the particles. In the case of a metal, an effective attraction between the electrons arises through an interaction with phonons, the quantized vibrations of the underlying lattice formed by atoms.

A connection between the two approaches, the phenomenological GL theory and the microscopic BCS theory, was established in 1959 when Gor'kov 8] explained how, close to the critical temperature, the GL theory arises from the BCS model. A simplified version of his explanation was later given by de Gennes in his textbook 6]. The purpose of this paper is to give the first fully rigorous mathematical derivation of GL theory from the BCS model. In our approach it is not necessary to restrict attention to the particular interaction considered in the original BCS model; indeed, we allow for a large class of attractions among the particles. In particular, we allow for local two-body interactions, in contrast to the simpler (non-local rank one) interaction considered by BCS. Such local interactions are relevant for the description of cold gases of fermionic atoms, which are of great current interest. These atoms are electrically neutral, and the corresponding pairing mechanism is relevant for superfluidity rather than superconductivity.

The BCS model is a considerable simplification of the full many-body problem. In the latter a state is described by a complicated wave function of a macroscopic number of variables. In the BCS model, all the information about the system is contained in quantities depending on only two variables; the reduced one-particle density matrix $\gamma$, i.e., a positive trace class operator on the one-particle space, and the Cooper pair (two-particle) wave function $\alpha$, which is non-zero only below the critical temperature. The GL model is yet much simpler, as it describes the system by a single function $\psi$ of one variable only, which satisfies a non-linear second-order PDE, the GL equation. This function $\psi$ only describes macroscopic variations in the system, whereas the BCS states $\gamma$ and $\alpha$ exhibit both microscopic and macroscopic details. Hence GL theory represents a significant simplification as to the more complicated BCS theory. The relation of these three theories is analogous to atomic physics where quantum mechanics, Hartree-Fock theory and Thomas-Fermi theory are models of the same type of increasing simplicity. In contrast to the atomic case, where the Hartree-Fock approximation is mathematically well understood, it remains an open problem to rigorously establish the relation of the BCS model to the full many-body quantum-mechanical description.

The BCS theory may be thought of as a variational theory, which utilizes a special class of trial states known as quasi-free states. This point of view was emphasized by Leggett [18. The mathematical aspects of this theory in the translation-invariant case were studied in [5, 10, 13, 14, 15. In this paper we study the non-translationinvariant case, where weak external fields are present that vary only on the macroscopic scale. We show that close to the critical temperature $T_{c}$, where $\alpha$ is small, the macroscopic variations of $\alpha$ are correctly described by the GL theory. More precisely, we assume that the microscopic scale of the system is of order $h \ll 1$ relative to the macroscopic scale. Variations of the system on the macroscopic scale cause a relative change of the energy of order $h^{2}$; hence the external fields should change the energy to the same order. This change is correctly described by the GL theory to leading order in $h$ if $\left(T_{c}-T\right) / T_{c}$ is of the order $h^{2}$, with $T$ the actual temperature. We also prove that the GL wave function $\psi$ correctly describes the macroscopic behavior of the BCS state. The BCS Cooper pair wave function in 
this parameter regime equals

$$
\alpha(x, y)=\frac{1}{2}(\psi(h x)+\psi(h y)) \alpha_{0}(x-y)
$$

to leading order in $h$, where $\alpha_{0}$ is the unperturbed translation-invariant pair function. Equivalently, $\frac{1}{2}(\psi(h x)+\psi(h y))$ could be replaced by $\psi(h(x+y) / 2)$ to leading order. In particular, $\psi$ describes the center-of-mass motion of the BCS state. The role of $h$ as a semiclassical parameter can be understood as follows. If one rescales the arguments to macroscopic variables and thinks of $\alpha(\bar{x} / h, \bar{y} / h)$ as the integral kernel of an operator, it is the quantization of the semiclassical symbol $\psi(\bar{x}) \widehat{\alpha}_{0}(p)$. It would, in fact, be the Weyl quantization if we had used the alternative centerof-mass representation. For technical reasons we found it more convenient to work with the representation (1.1).

Our approach is motivated by the work of de Gennes [6]. While de Gennes studied the emergence of the GL equation from the BCS equations, it is important for our rigorous analysis to utilize that these equations are Euler-Lagrange equations for variational problems. We give precise bounds on the lowest energy of the BCS functional and the connection of the corresponding minimizer with the GL minimizer. The exact statement of our result is given in Subsection 1.4 .

1.2. The BCS functional. We consider a macroscopic sample of a fermionic system, in $d$ spatial dimensions, where $1 \leq d \leq 3$. Let $\mu \in \mathbb{R}$ denote the chemical potential and $T>0$ the temperature of the sample. The fermions interact through a local two-body potential $V$. In addition, they are subject to external electric and/or magnetic fields. Neutral atoms would not couple to these fields, of course, but there can be other forces, e.g., arising from rotation, with a similar mathematical description. In BCS theory the state of the system can be conveniently described in terms of a $2 \times 2$ operator-valued matrix

$$
\Gamma=\left(\begin{array}{cc}
\gamma & \alpha \\
\bar{\alpha} & 1-\bar{\gamma}
\end{array}\right)
$$

satisfying $0 \leq \Gamma \leq 1$ as an operator on $L^{2}\left(\mathbb{R}^{d}\right) \oplus L^{2}\left(\mathbb{R}^{d}\right) \cong L^{2}\left(\mathbb{R}^{d}\right) \otimes \mathbb{C}^{2}$. The bar denotes complex conjugation, i.e., $\bar{\alpha}$ has the integral kernel $\overline{\alpha(x, y)}$. In particular, $\Gamma$ is Hermitian, implying that $\gamma$ is Hermitian and $\alpha$ is symmetric, i.e., $\gamma(x, y)=\overline{\gamma(y, x)}$ and $\alpha(x, y)=\alpha(y, x)$. There are no spin variables in $\Gamma$. The full, spin-dependent Cooper pair wave function is the product of $\alpha$ with an antisymmetric spin singlet. This is why $\alpha$ itself is symmetric so that we obtain the antisymmetric fermionic character of the full, spin-dependent, pair wave function.

The general form of the BCS functional for the free energy of such a system is, in suitable units,

$$
\operatorname{Tr}\left[\left((-i \nabla+A(x))^{2}-\mu+W(x)\right) \gamma\right]-T S(\Gamma)+\int V(x-y)|\alpha(x, y)|^{2} d x d y .
$$

Here, $A$ is the magnetic vector potential, and $W$ is the external electric potential. The entropy equals

$$
S(\Gamma)=-\operatorname{Tr}[\Gamma \ln \Gamma],
$$

where the trace is now both over $\mathbb{C}^{2}$ and $L^{2}$. (The precise definition will be given in the paragraph below (1.4).) The BCS state of the system is a minimizer of this functional over all admissible states $\Gamma$. 
As explained in detail in [10, Appendix A], the BCS functional can be heuristically derived from the full many-body Hamiltonian via two steps of simplification. First, one considers only quasi-free states, and second one neglects the resulting direct and exchange term in the interaction energy. The latter terms are considered unimportant in the physically relevant parameter regimes [19].

We are interested in the effect of weak and slowly varying external fields, as already explained in the previous subsection. More precisely, $A(x)$ should be replaced by $h A(h x)$ and $W(x)$ by $h^{2} W(h x)$. In order to avoid having to introduce boundary conditions, we assume that the system is infinite and periodic with period $h^{-1}$, in all $d$ directions. In particular, $A$ and $W$ should be periodic. We also assume that the state $\Gamma$ is periodic. The aim then is to calculate the free energy per unit volume.

We find it convenient to do a rescaling and use macroscopic variables instead of the microscopic ones. The rescaled BCS functional has the form

$$
\begin{aligned}
\mathcal{F}^{\mathrm{BCS}}(\Gamma):= & \operatorname{Tr}\left[\left((-i h \nabla+h A(x))^{2}-\mu+h^{2} W(x)\right) \gamma\right]-T S(\Gamma) \\
& +\int_{\mathcal{C} \times \mathbb{R}^{d}} V\left(h^{-1}(x-y)\right)|\alpha(x, y)|^{2} d x d y,
\end{aligned}
$$

where $\mathcal{C}$ denotes the unit cube $[0,1]^{d}$, and $\operatorname{Tr}$ stands for the trace per unit volume. More precisely, if $B$ is a periodic operator (i.e., it commutes with translations by 1 in the $d$ coordinate directions), then $\operatorname{Tr} B$ equals, by definition, the (usual) trace of $\chi B$, with $\chi$ the characteristic function of $\mathcal{C}$. The location of the cube is obviously of no importance. It is not difficult to see that the trace per unit volume has the usual properties such as cyclicity, and standard inequalities such as Hölder's inequality hold. This is discussed in more detail in Section 3 .

We make the following assumptions on the functions $A, W$ and $V$ appearing in (1.4).

Assumption 1. We assume both $W$ and $A$ to be periodic with period 1 . We further assume that $\widehat{W}(p)$ and $|\widehat{A}(p)|(1+|p|)$ are summable, with $\widehat{W}(p)$ and $\widehat{A}(p)$ denoting the Fourier coefficients of $W$ and $A$, respectively. In particular, $W$ is bounded and continuous and $A$ is in $C^{1}\left(\mathbb{R}^{d}\right)$.

The interaction potential $V$ is assumed to be real-valued and reflection-symmetric, i.e., $V(x)=V(-x)$, with $V \in L^{p}\left(\mathbb{R}^{d}\right)$, where $p=1$ for $d=1, p>1$ for $d=2$ and $p=3 / 2$ for $d=3$.

Our results presumably hold under slightly weaker regularity assumptions on $W$ and $A$. For the sake of transparency we shall not aim for the weakest possible conditions, but rather try to keep the proofs to a reasonable length.

The $L^{p}$ assumption on $V$ is the natural one to guarantee relative form-boundedness with respect to the Laplacian. In particular, the first term in (1.4) dominates the last term and also the entropy $S(\Gamma)$, and hence $\mathcal{F}^{\mathrm{BCS}}$ is bounded below. A slower decay of $V$ at infinity could possibly be accommodated. Our method also works for non-local potentials, which appear naturally in the theory of superconductivity. For simplicity, we are not aiming for the most general setting and work with Assumption 1 from now on.

We note that a periodic magnetic field $B$, satisfying Maxwell's equation $\nabla \cdot B=0$, can be described via a periodic vector potential $A$ as $B=\nabla \wedge A$ if certain flux conditions are satisfied. For $d=2, B$ has to have zero average, while for $d=3$ the flux through the boundaries of the unit cell has to vanish. This follows from the 
fact that the relevant de Rham cohomology (closed two-forms modulo exact ones) equals $\mathbb{R}^{n(n-1) / 2}$ for the $n$-dimensional torus.

1.2.1. The translation-invariant case. In the translation-invariant case, where $W=$ $A=0$, it makes sense to restrict $\mathcal{F}^{\mathrm{BCS}}$ to translation-invariant states $\Gamma$. This is the case studied in detail in 10.1 In particular, it was shown in [10 that there is a critical temperature $T_{c} \geq 0$ such that for $T \geq T_{c}$, the BCS functional $\mathcal{F}^{\mathrm{BCS}}$ is minimized for $\alpha=0$ and $\gamma=\left(1+\exp \left(\left(-h^{2} \nabla^{2}-\mu\right) / T\right)\right)^{-1}$; i.e., $\gamma$ is the one-particle density matrix of a free Fermi gas. The critical temperature $T_{c}$ is determined by the unique value of $T$ such that the operator

$$
K_{T}+V(x)
$$

on $L_{\text {sym }}^{2}\left(\mathbb{R}^{d}\right)$, the reflection-symmetric square-integrable functions, has zero as its lowest eigenvalue 2 Here, $K_{T}$ denotes the operator

$$
K_{T}=\frac{-\nabla^{2}-\mu}{\tanh \left(\frac{-\nabla^{2}-\mu}{2 T}\right)} .
$$

Note that $K_{T}$ is monotone increasing in $T$ and $K_{T} \geq 2 T$. (If $K_{0}+V=\left|-\nabla^{2}-\mu\right|+$ $V \geq 0$, then $T_{c}=0$.) In particular, the essential spectrum of $K_{T}+V$ is $[2 T, \infty)$, and hence an eigenvalue at 0 is necessarily isolated.

In the following, we shall assume that $T_{c}>0$ and that the ground state of (1.5) at $T=T_{c}$ is non-degenerate. We emphasize that $T_{c}$ is independent of $h$.

Assumption 2. The potential $V$ is such that $T_{c}>0$ and that $K_{T_{c}}+V$ has a non-degenerate ground state eigenvalue 0 , whose corresponding eigenfunction will be denoted by $\alpha_{0}$.

According to 13 this assumption is satisfied if $\widehat{V} \leq 0$ (and not identically zero), for instance. In the case that $V$ is invariant under rotations, the non-degeneracy assumption means that the minimizing function has angular momentum zero.

Important properties of $\alpha_{0}$, which will be used in the proof of our main results, are collected in Appendix $\mathrm{A}$.

1.3. The GL functional. Let $\psi \in H_{\text {per }}^{1}\left(\mathbb{R}^{d}\right)$, the periodic functions in $H_{\mathrm{loc}}^{1}\left(\mathbb{R}^{d}\right)$. For $\mathbb{B}_{1}$ a positive $d \times d$ matrix, $B_{2} \in \mathbb{R}$ and $B_{3}>0$, the GL functional is defined as

$$
\begin{array}{r}
\mathcal{E}^{\mathrm{GL}}(\psi, A)=\int_{\mathcal{C}}\left[(\overline{(-i \nabla+2 A(x)) \psi}) \cdot \mathbb{B}_{1}((-i \nabla+2 A(x)) \psi)\right. \\
\left.+B_{2} W(x)|\psi(x)|^{2}+\left.\left.B_{3}|1-| \psi(x)\right|^{2}\right|^{2}\right] d x .
\end{array}
$$

Note the coefficient 2 in front of the vector potential $A$. It is due to the fact that $\psi$ describes pairs of particles, and the charge of a pair is twice the particle charge. The relevant coefficients $\mathbb{B}_{1}, B_{2}$ and $B_{3}$ will be calculated below from the BCS theory; see Eqs. (1.19) -(1.21). In the rotation-invariant case (i.e., for radial potentials $V$ ), the matrix $\mathbb{B}_{1}$ is a multiple of the identity matrix.

The ground state energy of the GL functional will be denoted by

$$
E^{\mathrm{GL}}=\inf \left\{\mathcal{E}(\psi): \psi \in H_{\mathrm{per}}^{1}\left(\mathbb{R}^{d}\right)\right\} .
$$

\footnotetext{
${ }^{1}$ The results in [10] are worked out in three dimensions, but analogous results are easily seen to hold in one and two dimensions.

${ }^{2}$ In [10], the symmetry constraint on $\alpha$ was not explicitly enforced. The results hold equally if one works only in the subspace of reflection-symmetric functions in $L^{2}\left(\mathbb{R}^{d}\right)$, however.
} 
It is not difficult to show that under our assumptions on $A$ and $W$, there exists a corresponding minimizer, which satisfies a second-order differential equation known as the GL equation. The mathematical aspects of the GL functional have been studied extensively in the literature; see [4, 9, 24] and the references therein.

1.4. Main results. Recall the definition of the BCS functional $\mathcal{F}^{\mathrm{BCS}}$ in (1.4). Recall also that admissible states $\Gamma$ are of the form (1.2), are periodic with period 1 and with $0 \leq \Gamma \leq 1$. We define the energy $F^{\mathrm{BCS}}(T, \mu)$ as the difference between the infimum of $\mathcal{F}^{\mathrm{BCS}}$ over all admissible $\Gamma$ and the free energy of the normal state

$$
\Gamma_{0}:=\left(\begin{array}{cc}
\gamma_{0} & 0 \\
0 & 1-\bar{\gamma}_{0}
\end{array}\right)
$$

where $\gamma_{0}=\left(1+e^{\left((-i h \nabla+h A(x))^{2}+h^{2} W(x)-\mu\right) / T}\right)^{-1}$. I.e.,

$$
F^{\mathrm{BCS}}(T, \mu)=\inf _{\Gamma} \mathcal{F}^{\mathrm{BCS}}(\Gamma)-\mathcal{F}^{\mathrm{BCS}}\left(\Gamma_{0}\right) .
$$

Note that $\Gamma_{0}$ is the minimizer of the BCS functional in the absence of interactions, i.e., when $V=0$. We have

$$
\mathcal{F}^{\mathrm{BCS}}\left(\Gamma_{0}\right)=-T \operatorname{Tr} \ln \left(1+\exp \left(-\left((-i h \nabla+h A(x))^{2}-\mu+h^{2} W(x)\right) / T\right)\right),
$$

which is $O\left(h^{-d}\right)$ for small $h$. The system is said to be in a superconducting (or superfluid, depending on the physical interpretation) state if $F^{\mathrm{BCS}}<0$.

Theorem 1. As above, let $T_{c}>0$ denote the critical temperature in the translationinvariant case, and assume Assumptions 1 and 2. Let $D>0$. For appropriate coefficients $\mathbb{B}_{1}, B_{2}$ and $B_{3}$ (which will be explicitly given in (1.19)-(1.21) below), we have, as $h \rightarrow 0$,

$$
F^{\mathrm{BCS}}\left(T_{c}\left(1-h^{2} D\right), \mu\right)=h^{4-d}\left(E^{\mathrm{GL}}-B_{3}+e\right),
$$

where $E^{\mathrm{GL}}$ is defined in (1.7)-(1.8) and e satisfies the bounds

$$
\text { const } h \geq e \geq-e_{\mathrm{L}}:=- \text { const } \times\left\{\begin{array}{cl}
h^{1 / 3} & \text { for } d=1, \\
h^{1 / 3}[\ln (1 / h)]^{1 / 6} & \text { for } d=2, \\
h^{1 / 5} & \text { for } d=3 .
\end{array}\right.
$$

Moreover, if $\Gamma$ is an approximate minimizer of $\mathcal{F}^{\mathrm{BCS}}$ at $T=T_{c}\left(1-h^{2} D\right)$, in the sense that

$$
\mathcal{F}^{\mathrm{BCS}}(\Gamma) \leq \mathcal{F}^{\mathrm{BCS}}\left(\Gamma_{0}\right)+h^{4-d}\left(E^{\mathrm{GL}}-B_{3}+\epsilon\right)
$$

for some small $\epsilon>0$, then the corresponding $\alpha$ can be decomposed as

$$
\alpha=\frac{h}{2}\left(\psi(x) \widehat{\alpha}_{0}(-i h \nabla)+\widehat{\alpha}_{0}(-i h \nabla) \psi(x)\right)+\sigma
$$

with the function $\psi$ satisfying the estimate $\mathcal{E}^{\mathrm{GL}}(\psi) \leq E^{\mathrm{GL}}+\epsilon+e_{\mathrm{L}}, \alpha_{0}$ the (appropriately normalized; see (1.18) ) zero-energy ground state of (1.5) at $T=T_{c}$, and

$$
\int_{\mathcal{C} \times \mathbb{R}^{d}}|\sigma(x, y)|^{2} d x d y \leq \text { const } \frac{h^{4-d}}{e_{\mathrm{L}}^{2}} .
$$


To appreciate the bound (1.16), note that the square of the $L^{2}\left(\mathcal{C} \times \mathbb{R}^{d}\right)$ norm of the first term on the right side of (1.15) is of the order $h^{2-d}$, and hence is much larger than the one of $\sigma$ for small $h$. Theorem 1 thus justifies the claim made in the Introduction in (1.1).

For smooth enough $A$ and $W$, one could also expand $\mathcal{F}^{\mathrm{BCS}}\left(\Gamma_{0}\right)$ in (1.11) to order $h^{4-d}$ and thus obtain the total energy $\inf _{\Gamma} \mathcal{F}^{\mathrm{BCS}}(\Gamma)$ to this order. Our approach bounds directly the difference (1.10), however, and hence it is not necessary to compute $\mathcal{F}^{\mathrm{BCS}}\left(\Gamma_{0}\right)$ in detail, and to make the corresponding additional regularity assumptions on $A$ and $W$.

Our proof shows that the coefficients $\mathbb{B}_{1}, B_{2}$ and $B_{3}$ in the GL functional are given as follows. Recall that $\alpha_{0}$ denotes the unique ground state of $K_{T_{c}}+V$. It satisfies $\alpha_{0}(x)=\alpha_{0}(-x)$, and we can take it to be real. Let $t$ denote the Fourier transform of $2 K_{T_{c}} \alpha_{0}=-2 V \alpha_{0}$, i.e.,

$$
t(p)=-2(2 \pi)^{-d / 2} \int_{\mathbb{R}^{d}} V(x) \alpha_{0}(x) e^{-i p \cdot x} d x .
$$

We normalize it, i.e., choose the normalization of $\alpha_{0}$, such that

$$
\int_{\mathbb{R}^{d}} t(q)^{4} \frac{g_{1}\left(\beta_{c}\left(q^{2}-\mu\right)\right)}{q^{2}-\mu} d q=\frac{D}{\beta_{c}} \int_{\mathbb{R}^{d}} t(q)^{2} \cosh ^{-2}\left(\frac{\beta_{c}}{2}\left(q^{2}-\mu\right)\right) d q,
$$

where $\beta_{c}=1 / T_{c}, D=\left(1-T / T_{c}\right) / h^{2}$ as above, and $g_{1}$ denotes the function

$$
g_{1}(z)=\frac{e^{2 z}-2 z e^{z}-1}{z^{2}\left(1+e^{z}\right)^{2}} .
$$

Note that $g_{1}(z) / z>0$. Define also

$$
g_{2}(z)=\frac{2 e^{z}\left(e^{z}-1\right)}{z\left(e^{z}+1\right)^{3}}
$$

(compare with (2.8)-(2.11) ). Then $\mathbb{B}_{1}$ is the matrix with components

$$
\left(\mathbb{B}_{1}\right)_{i j}=\frac{\beta_{c}^{2}}{16} \int_{\mathbb{R}^{d}} t(q)^{2}\left(\delta_{i j} g_{1}\left(\beta_{c}\left(q^{2}-\mu\right)\right)+2 \beta_{c} q_{i} q_{j} g_{2}\left(\beta_{c}\left(q^{2}-\mu\right)\right)\right) \frac{d q}{(2 \pi)^{d}} .
$$

Note that in case $V$ is radial, also $\alpha_{0}$ and $t$ are radial and thus $\mathbb{B}_{1}$ is proportional to the identity matrix. Moreover, $B_{2}$ and $B_{3}$ are given by

$$
B_{2}=\frac{\beta_{c}^{2}}{4} \int_{\mathbb{R}^{d}} t(q)^{2} g_{1}\left(\beta_{c}\left(q^{2}-\mu\right)\right) \frac{d q}{(2 \pi)^{d}}
$$

and

$$
B_{3}=\frac{\beta_{c}^{2}}{16} \int_{\mathbb{R}^{d}} t(q)^{4} \frac{g_{1}\left(\beta_{c}\left(q^{2}-\mu\right)\right)}{q^{2}-\mu} \frac{d q}{(2 \pi)^{d}},
$$

respectively. Alternatively, $B_{3}$ could be written using the normalization (1.18). In particular, $B_{3} /\left|B_{2}\right|$ and $B_{3} /\left\|\mathbb{B}_{1}\right\|$ are proportional to $D$, and hence proportional to the difference of the temperature to the critical one.

Note that $B_{3}>0$ since $g_{1}(z) / z>0$ for all $z \in \mathbb{R}$. The coefficient $B_{2}$ can, in principle, have either sign if $\mu>0$, however. It has the same sign as the derivative of $T_{c}$ with respect to $\mu$. This is not surprising. The external potential plays the role of a local variation in the chemical potential. If increasing $\mu$ increases $T_{c}$, the pairing mechanism should be enhanced at negative values of $W$ and the density of Cooper pairs should be largest there. If increasing $\mu$ decreases $T_{c}$, however, the situation is reversed. 
To see that the matrix $\mathbb{B}_{1}$ is positive, we calculate

$$
\begin{aligned}
& 2 \operatorname{Re}\left\langle\alpha_{0}\left|x_{i}\left(K_{T_{c}}+V\right) x_{j}\right| \alpha_{0}\right\rangle=\left\langle\alpha_{0}\left|\left[x_{i},\left[K_{T_{c}}, x_{j}\right]\right]\right| \alpha_{0}\right\rangle \\
& =8(2 \pi)^{d} \mathbb{B}_{1, i j}-2 \beta_{c}^{3} \int_{\mathbb{R}^{d}} t(q)^{2} q_{i} q_{j} \frac{g_{1}\left(\beta_{c}\left(q^{2}-\mu\right)\right)}{\sinh \left(\beta_{c}\left(p^{2}-\mu\right)\right)} d q .
\end{aligned}
$$

The last term on the right side defines a negative matrix, while the left side defines a positive matrix, since $K_{T_{c}}+V \geq 0$. Hence $\mathbb{B}_{1}$ is positive.

1.5. Outline of the paper. The structure of the remainder of this paper is as follows. In Section 2 we shall state our main semiclassical estimates. These are a crucial input to obtain the bounds in Theorem 1. The leading terms in our semiclassical expansion can, in principle, be obtained from well-known formulas in semiclassical analysis, but the standard techniques do not apply directly because we are forced to work with rather minimal regularity assumptions. We shall formulate the main results in separate theorems; see Theorems 2 and 3 below. Their proofs, which are rather lengthy, will be given in Sections 7 and 8 , respectively. Some technicalities are deferred to Appendix B

Section 3 explains various inequalities for the trace per unit volume which will be used throughout the proofs. An upper bound on $F^{\mathrm{BCS}}$ will be derived in Section 4, using the variational principle. An important input will be the semiclassical estimates of Theorems 2 and 3 .

Sections 5 and 6 contain the lower bound. In the first part, the structure of an approximate minimizer is investigated, which leads to a definition of the order parameter $\psi$. This structure is then a crucial input to the second part, where also the semiclassical estimates of Section 2 enter.

Properties of $\alpha_{0}$, the ground state of (1.5) at $T=T_{c}$, are derived in Appendix A It is shown that $t$, defined in (1.17) above, is smooth and has a suitable decay at infinity. The results imply, in particular, that the coefficients (1.19)-(1.21) are well-defined and finite.

Throughout the proofs, $C$ will denote various different constants. We will sometimes be sloppy and use $C$ also for expressions that depend only on some fixed, $h$-independent, quantities such as $\mu, T_{c}$ or $\|W\|_{\infty}$, for instance.

\section{Semiclassical estimates}

One of the key ingredients in the proof of Theorem 1 is semiclassical analysis. Choose a periodic function $\psi \in H_{\mathrm{loc}}^{2}\left(\mathbb{R}^{d}\right)$ and a sufficiently nice function $t$ and let $\Delta$ be the operator

$$
\Delta=-\frac{h}{2}(\psi(x) t(-i h \nabla)+t(-i h \nabla) \psi(x)) .
$$

It has the integral kernel

$$
\Delta(x, y)=-\frac{h^{1-d}}{2(2 \pi)^{d / 2}}(\psi(x)+\psi(y)) \widehat{t}\left(h^{-1}(y-x)\right) .
$$

Our convention for the Fourier transform is that $\widehat{f}(p)=(2 \pi)^{-d / 2} \int_{\mathbb{R}^{d}} f(x) e^{-i p \cdot x} d x$. We shall assume that

$$
\partial^{\gamma} t \in L^{2 p /(p-1)}\left(\mathbb{R}^{d}\right)
$$


(with $p$ defined in Assumption 1) and that

$$
\int_{\mathbb{R}^{d}} \frac{\left|\partial^{\gamma} t(q)\right|^{2}}{1+q^{2}} d q<\infty
$$

for all $\gamma \in\{0,1, \ldots, 4\}^{d}$. For simplicity, we also assume that $t$ is reflectionsymmetric and real-valued. For the function $t$ in (1.17), these assumptions are satisfied, as will be shown in Appendix A

Let $H_{\Delta}$ be the operator

$$
H_{\Delta}=\left(\begin{array}{cc}
(-i h \nabla+h A(x))^{2}-\mu+h^{2} W(x) & \Delta \\
\bar{\Delta} & -(i h \nabla+h A(x))^{2}+\mu-h^{2} W(x)
\end{array}\right)
$$

on $L^{2}\left(\mathbb{R}^{d}\right) \otimes \mathbb{C}^{2}$, with $A$ and $W$ satisfying Assumption 1 . In the following, we will investigate the trace per unit volume of functions of $H_{\Delta}$. Specifically, we are interested in the effect of the off-diagonal term $\Delta$ in $H_{\Delta}$, in the semiclassical regime of small $h$. The functions of $H_{\Delta}$ we are considering are not actually locally trace class, in general, but their diagonal entries are. We are thus led to define

$$
\operatorname{Tr}_{0} O=\operatorname{Tr}\left[P_{0} O P_{0}+Q_{0} O Q_{0}\right]
$$

with

$$
P_{0}=\left(\begin{array}{ll}
1 & 0 \\
0 & 0
\end{array}\right)
$$

and $Q_{0}=1-P_{0}$. Note that if $O$ is locally trace class, then $\operatorname{Tr}_{0} O=\operatorname{Tr} O$. This identity also holds for all non-negative operators $O$, in the sense that either both sides are infinite or otherwise equal.

Theorem 2. Let

$$
f(z)=-\ln \left(1+e^{-z}\right)
$$

and define

$$
\begin{gathered}
g_{0}(z)=\frac{f^{\prime}(-z)-f^{\prime}(z)}{z}=\frac{\tanh \left(\frac{1}{2} z\right)}{z} \\
g_{1}(z)=-g_{0}^{\prime}(z)=\frac{f^{\prime}(-z)-f^{\prime}(z)}{z^{2}}+\frac{f^{\prime \prime}(-z)+f^{\prime \prime}(z)}{z}=\frac{e^{2 z}-2 z e^{z}-1}{z^{2}\left(1+e^{z}\right)^{2}}
\end{gathered}
$$

and

$$
g_{2}(z)=g_{1}^{\prime}(z)+\frac{2}{z} g_{1}(z)=\frac{f^{\prime \prime \prime}(z)-f^{\prime \prime \prime}(-z)}{z}=\frac{2 e^{z}\left(e^{z}-1\right)}{z\left(e^{z}+1\right)^{3}} .
$$

Then, for any $\beta>0$, the diagonal entries of the $2 \times 2$ matrix-valued operator $f\left(\beta H_{\Delta}\right)-f\left(\beta H_{0}\right)$ are locally trace class, and the sum of their traces per unit volume equals

$$
\begin{aligned}
\frac{h^{d}}{\beta} \operatorname{Tr}_{0}\left[f\left(\beta H_{\Delta}\right)-f\left(\beta H_{0}\right)\right]= & h^{2} E_{1}+h^{4} E_{2}+O\left(h^{5}\right)\left(\|\psi\|_{H^{1}(\mathcal{C})}^{4}+\|\psi\|_{H^{1}(\mathcal{C})}^{2}\right) \\
& +O\left(h^{6}\right)\left(\|\psi\|_{H^{1}(\mathcal{C})}^{6}+\|\psi\|_{H^{2}(\mathcal{C})}^{2}\right),
\end{aligned}
$$

where

$$
E_{1}=-\frac{\beta}{2}\|\psi\|_{2}^{2} \int_{\mathbb{R}^{d}} t(q)^{2} g_{0}\left(\beta\left(q^{2}-\mu\right)\right) \frac{d q}{(2 \pi)^{d}}
$$


and

$$
\begin{aligned}
E_{2}= & -\frac{\beta}{8} \sum_{j, k=1}^{d}\left\langle\partial_{j} \psi \mid \partial_{k} \psi\right\rangle \int_{\mathbb{R}^{d}} t(q)\left[\partial_{j} \partial_{k} t\right](q) g_{0}\left(\beta\left(q^{2}-\mu\right)\right) \frac{d q}{(2 \pi)^{d}} \\
& +\frac{\beta^{2}}{8} \sum_{j, k=1}^{d}\left\langle\left(\partial_{j}+2 i A_{j}\right) \psi \mid\left(\partial_{k}+2 i A_{k}\right) \psi\right\rangle \\
& \times \int_{\mathbb{R}^{d}} t(q)^{2}\left(\delta_{j k} g_{1}\left(\beta\left(q^{2}-\mu\right)\right)+2 \beta q_{j} q_{k} g_{2}\left(\beta\left(q^{2}-\mu\right)\right)\right) \frac{d q}{(2 \pi)^{d}} \\
& +\frac{\beta^{2}}{2}\langle\psi|W| \psi\rangle \int_{\mathbb{R}^{d}} t(q)^{2} g_{1}\left(\beta\left(q^{2}-\mu\right)\right) \frac{d q}{(2 \pi)^{d}} \\
& +\frac{\beta^{2}}{8}\|\psi\|_{4}^{4} \int_{\mathbb{R}^{d}} t(q)^{4} \frac{g_{1}\left(\beta\left(q^{2}-\mu\right)\right)}{q^{2}-\mu} \frac{d q}{(2 \pi)^{d}} .
\end{aligned}
$$

The error terms in (2.12) of order $h^{5}$ and $h^{6}$ are bounded uniformly in $\beta$ for $\beta$ in compact intervals in $(0, \infty)$. They depend on $t$ only via bounds on the expressions (2.3) and (2.4).

Here, we use the short-hand notation $\|\psi\|_{p}$ for the norm on $L^{p}(\mathcal{C})$. Likewise, $\langle\cdot \mid \cdot\rangle$ denotes the inner product on $L^{2}(\mathcal{C})$.

In general, the operator $f\left(\beta H_{\Delta}\right)-f\left(\beta H_{0}\right)$ will not be locally trace class under our assumptions on $t$ and $\psi$. Hence the trace in (2.12) has to be suitably understood as the sum of the traces of the diagonal entries, defined in (2.6). This issue is further discussed in Section 4 .

The expressions $E_{1}$ and $E_{2}$ are the first two non-vanishing terms in a semiclassical expansion of the left side of (2.12). They can be obtained, in principle, from wellknown formulas in semiclassical analysis [17, 23]. The standard techniques are not directly applicable in our case, however. This has to do, on the one hand, with our rather minimal regularity assumptions on $W, A, \psi$ and $t$ and, on the other hand, with the fact that we are working with the trace per unit volume of an infinite, periodic system.

The proof of Theorem 2 will be given in Section 7 As the proof shows, the theorem holds for a larger class of functions $f$, satisfying appropriate smoothness and decay assumptions.

If $\mu<0$, the bounds in Theorem 2 are actually uniform in the temperature $\beta^{-1}$ even as $\beta^{-1} \rightarrow 0$. This observation was used in [16] to study a system of fermions at zero temperature in the low density limit.

Our second semiclassical estimate concerns the upper off-diagonal term of

$$
\Gamma_{\Delta}=\frac{1}{1+e^{\beta H_{\Delta}}},
$$

which we shall denote by $\alpha_{\Delta}$. We shall be interested in its $H^{1}$-norm. In general, we define the $H^{1}$-norm of a periodic operator $O$ by

$$
\|O\|_{H^{1}}^{2}=\operatorname{Tr}\left[O^{\dagger}\left(1-h^{2} \nabla^{2}\right) O\right] .
$$

In other words, $\|O\|_{H^{1}}^{2}=\|O\|_{2}^{2}+h^{2}\|\nabla O\|_{2}^{2}$. Note that this definition is not symmetric, i.e., $\|O\|_{H^{1}} \neq\left\|O^{\dagger}\right\|_{H^{1}}$ in general. 
Given $t$ as above, let us define the function

$$
\varphi(p)=\frac{\beta}{2} g_{0}\left(\beta\left(p^{2}-\mu\right)\right) t(p) .
$$

Theorem 3. With $\alpha_{\Delta}$ denoting the upper off-diagonal entry of $\Gamma_{\Delta}$ in (2.15), we have

$$
\left\|\alpha_{\Delta}-\frac{h}{2}(\psi(x) \varphi(-i h \nabla)+\varphi(-i h \nabla) \psi(x))\right\|_{H^{1}} \leq C h^{3-d / 2}\left(\|\psi\|_{H^{2}(\mathcal{C})}+\|\psi\|_{H^{1}(\mathcal{C})}^{3}\right) \text {. }
$$

The proof of this theorem will be given in Section 8 .

\section{Preliminaries}

In this section we collect a few useful facts about the trace per unit volume. In particular, we recall the general form of Hölder's inequality and Klein's inequality, both of which will be used several times in the proofs below.

Let $A$ and $B$ be bounded periodic operators on either $L^{2}\left(\mathbb{R}^{d}\right)$ or $L^{2}\left(\mathbb{R}^{d} ; \mathbb{C}^{2}\right)$, i.e., operators that commute with translations by a unit length in any of the $d$ coordinate directions. The trace per unit volume of $A$ is simply defined as the trace of $\chi A \chi$, where $\chi$ is the characteristic function of a unit cube, i.e., the projection onto functions supported in this cube. Obviously, the location of the cube is irrelevant. For $p \geq 1$ we also denote the $p$-norm of $A$ by

$$
\|A\|_{p}=\left(\operatorname{Tr}\left(A^{\dagger} A\right)^{p / 2}\right)^{1 / p} .
$$

Here and in the remainder of this paper, Tr denotes the trace per unit volume. We also use the notation $\|A\|_{\infty}$ for the standard operator norm.

For any $1 \leq p \leq \infty$, the triangle inequality

$$
\|A+B\|_{p} \leq\|A\|_{p}+\|B\|_{p}
$$

holds. For $1 / r+1 / s=1 / p, 1 \leq r, s, p \leq \infty$, we have the general Hölder inequality

$$
\|A B\|_{p} \leq\|A\|_{r}\|B\|_{s} .
$$

Moreover, if $f: \mathbb{R} \times \mathbb{R} \rightarrow \mathbb{R}_{+}$is a non-negative function of the form $f(x, y)=$ $\sum_{i} g_{i}(x) h_{i}(y)$, then Klein's inequality

$$
\operatorname{Tr} f(A, B):=\sum_{i} \operatorname{Tr} g_{i}(A) h_{i}(B) \geq 0
$$

holds for selfadjoint $A$ and $B$. The same is true with $\operatorname{Tr}$ replaced by $\operatorname{Tr}_{0}$ (defined in (2.6) ) if either $A$ or $B$ commutes with $P_{0}$.

Inequalities (3.2)-3.4) are well known in the case of standard traces; see 25. and 26]. They extend to the periodic case via the Floquet decomposition [22, Sect. XIII.16]. Specifically,

$$
A \cong \int_{[0,2 \pi]^{d}}^{\oplus} A^{\xi} \frac{d \xi}{(2 \pi)^{d}}
$$

with $A^{\xi}$ operating on $L^{2}(\mathcal{C})$, and $\cong$ denotes unitary equivalence. The trace per unit volume equals

$$
\operatorname{Tr} A=\int_{[0,2 \pi]^{d}} \operatorname{Tr}_{L^{2}(\mathcal{C})} A^{\xi} \frac{d \xi}{(2 \pi)^{d}} .
$$


The inequalities (3.2)-(3.4) then easily follow from the standard ones using that

$$
(A B)^{\xi}=A^{\xi} B^{\xi}
$$

and that $g(A)^{\xi}=g\left(A^{\xi}\right)$ for appropriate functions $g$ and selfadjoint $A$.

One also checks that

$$
|\operatorname{Tr} A| \leq\|A\|_{1}
$$

By induction, if follows from (3.3) that

$$
\left|\operatorname{Tr} A_{1} A_{2} \cdots A_{n}\right| \leq\left\|A_{1} A_{2} \cdots A_{n}\right\|_{1} \leq \prod_{i=1}^{n}\left\|A_{i}\right\|_{p_{i}}
$$

where $1 \leq p_{i} \leq \infty$ and $\sum_{i} p_{i}^{-1}=1$.

Note that while the local trace norms introduced here share many properties with the usual Schatten norms, they are not monotone decreasing in $p$. For instance, if $A^{\xi}$ is a rank-one operator (whose norm is not independent of $\xi$ ), then the norm $\|A\|_{p}$ is actually increasing in $p$. In particular, the finiteness of the $p$-norm does not, in general, imply finiteness of the $q$-norm for $q>p$.

In the proofs below we need one more inequality that generalizes an inequality by Lieb and Thirring to the case of the trace per unit volume (see, e.g., [21, Sect. 4.5]). If $A$ and $B$ are periodic operators and $p \geq 2$, then

$$
\|A B\|_{p}^{p}=\operatorname{Tr}|A B|^{p}=\operatorname{Tr}\left(\left|B^{\dagger}\right||A|^{2}\left|B^{\dagger}\right|\right)^{p / 2} \leq \operatorname{Tr}\left|B^{\dagger}\right|^{p / 2}|A|^{p}\left|B^{\dagger}\right|^{p / 2} .
$$

Again, the proof follows from the standard one using the Floquet decomposition. In the special case that $A$ is a multiplication by a periodic function $a(x)$ and $B$ is multiplication in Fourier space, i.e., $B=b(-i \nabla)$, (3.10) reads

$$
\|a(x) b(-i \nabla)\|_{p} \leq(2 \pi)^{-d / p}\|a\|_{L^{p}(\mathcal{C})}\|b\|_{L^{p}\left(\mathbb{R}^{d}\right)} .
$$

\section{Proof of Theorem 1: Upper Bound}

In this section, we shall prove the upper bound in Theorem 1 i.e., we shall show that $e \leq C h$ with $e$ defined in (1.12). We shall denote $\beta=1 / T$ and $\beta_{c}=1 / T_{c}$. Recall that $\beta_{c} / \beta=1-h^{2} D$ with $D>0$.

By definition, $F^{\mathrm{BCS}}(T, \mu) \leq \mathcal{F}^{\mathrm{BCS}}(\Gamma)-\mathcal{F}^{\mathrm{BCS}}\left(\Gamma_{0}\right)$ for any admissible state $\Gamma$. As a trial state, we use

$$
\Gamma_{\Delta}=\left(\begin{array}{cc}
\gamma_{\Delta} & \alpha_{\Delta} \\
\bar{\alpha}_{\Delta} & 1-\bar{\gamma}_{\Delta}
\end{array}\right)=\frac{1}{1+e^{\beta H_{\Delta}}},
$$

where $H_{\Delta}$ is given in (2.5) with $\Delta$ as in (2.1). Note that $\Gamma_{0}$, defined in (1.9), indeed corresponds to setting $\Delta=0$ in (4.1). For $t$, we choose (1.17), which is reflection-symmetric and can be taken to be real. We normalize it such that (1.18) holds. The integral kernel of $\Delta$ is then given by

$$
\Delta(x, y)=\frac{h^{1-d}}{(2 \pi)^{d / 2}}(\psi(x)+\psi(y)) V\left(h^{-1}(x-y)\right) \alpha_{0}\left(h^{-1}(x-y)\right) .
$$

Note that $H_{\Delta}$ is unitarily equivalent to $-\bar{H}_{\Delta}$,

$$
U H_{\Delta} U^{\dagger}=-\bar{H}_{\Delta} \quad \text { with } \quad U=\left(\begin{array}{cc}
0 & 1 \\
-1 & 0
\end{array}\right) .
$$

Hence also $U \Gamma_{\Delta} U^{\dagger}=1-\bar{\Gamma}_{\Delta}$ and, in particular,

$$
S\left(\Gamma_{\Delta}\right)=-\frac{1}{2} \operatorname{Tr}\left[\Gamma_{\Delta} \ln \Gamma_{\Delta}+\left(1-\Gamma_{\Delta}\right) \ln \left(1-\Gamma_{\Delta}\right)\right] .
$$


Here, $\operatorname{Tr}$ could as well be replaced by $\operatorname{Tr}_{0}$, the sum of the traces per unit volume of the diagonal entries of a $2 \times 2$ matrix-valued operator defined in (2.6), since the operator in question is negative. A simple calculation shows that

$$
\begin{aligned}
& \Gamma_{\Delta} \ln \Gamma_{\Delta}+\left(1-\Gamma_{\Delta}\right) \ln \left(1-\Gamma_{\Delta}\right)-\Gamma_{0} \ln \Gamma_{0}-\left(1-\Gamma_{0}\right) \ln \left(1-\Gamma_{0}\right) \\
& =-\beta H_{\Delta} \Gamma_{\Delta}+\beta H_{0} \Gamma_{0}-\ln \left(1+e^{-\beta H_{\Delta}}\right)+\ln \left(1+e^{-\beta H_{0}}\right) .
\end{aligned}
$$

Moreover,

$$
H_{\Delta} \Gamma_{\Delta}-H_{0} \Gamma_{0}=\left(\begin{array}{cc}
k\left(\gamma_{\Delta}-\gamma_{0}\right)+\Delta \bar{\alpha}_{\Delta} & k \alpha_{\Delta}+\Delta\left(1-\bar{\gamma}_{\Delta}\right) \\
\bar{\Delta} \gamma_{\Delta}+\bar{k} \bar{\alpha}_{\Delta} & \bar{k}\left(\bar{\gamma}_{\Delta}-\bar{\gamma}_{0}\right)+\bar{\Delta} \alpha_{\Delta}
\end{array}\right)
$$

where $k$ denotes the left upper entry of $H_{\Delta}\left(\right.$ and $\left.H_{0}\right)$. From (1.4) and (4.4)-(4.6) we conclude that

$$
\begin{aligned}
\mathcal{F}^{\mathrm{BCS}}\left(\Gamma_{\Delta}\right)-\mathcal{F}^{\mathrm{BCS}}\left(\Gamma_{0}\right) \\
=-\frac{1}{2 \beta} \operatorname{Tr}_{0}\left[\ln \left(1+e^{-\beta H_{\Delta}}\right)-\ln \left(1+e^{-\beta H_{0}}\right)\right] \\
\quad-h^{2-2 d} \int_{\mathcal{C} \times \mathbb{R}^{d}} V\left(\frac{x-y}{h}\right)\left|\frac{1}{2}(\psi(x)+\psi(y)) \alpha_{0}\left(\frac{x-y}{h}\right)\right|^{2} \frac{d x d y}{(2 \pi)^{d}} \\
\quad+\int_{\mathcal{C} \times \mathbb{R}^{d}} V\left(\frac{x-y}{h}\right)\left|\frac{h^{1-d}}{2(2 \pi)^{d / 2}}(\psi(x)+\psi(y)) \alpha_{0}\left(\frac{x-y}{h}\right)-\alpha_{\Delta}(x, y)\right|^{2} d x d y .
\end{aligned}
$$

In general, the operator $\ln \left(1+e^{-\beta H_{\Delta}}\right)-\ln \left(1+e^{-\beta H_{0}}\right)$ is not locally trace class if $\Delta$ is not, as can be seen from (4.5)-(4.6). In the evaluation of $\mathcal{F}^{\mathrm{BCS}}\left(\Gamma_{\Delta}\right)-\mathcal{F}^{\mathrm{BCS}}\left(\Gamma_{0}\right)$ only the diagonal terms of (4.6) enter, however.

The first term on the right side of (4.7) was calculated in Theorem 2 above. Since $\beta=\beta_{c}+O\left(h^{2}\right)$, we can replace $\beta$ by $\beta_{c}$ in all the terms of order $h^{4}$, yielding an error of order $h^{6}$. For the term of order $h^{2}$, we obtain

$$
\begin{aligned}
& h^{2} \beta \int_{\mathbb{R}^{d}} t(q)^{2} g_{0}\left(\beta\left(q^{2}-\mu\right)\right) d q \\
& =h^{2} \beta_{c} \int_{\mathbb{R}^{d}} t(q)^{2} g_{0}\left(\beta_{c}\left(q^{2}-\mu\right)\right) d q \\
& \quad+h^{4} \beta_{c} D \int_{\mathbb{R}^{d}} t(q)^{2}\left(g_{0}\left(\beta_{c}\left(q^{2}-\mu\right)\right)-\beta_{c}\left(q^{2}-\mu\right) g_{1}\left(\beta_{c}\left(q^{2}-\mu\right)\right)\right) d q+O\left(h^{6}\right),
\end{aligned}
$$

where $D=\left(T_{c}-T\right) /\left(h^{2} T_{c}\right)$. The term in the last line of order $h^{4}$ equals

$$
\frac{h^{4} \beta_{c}}{2} D \int_{\mathbb{R}^{d}} t(q)^{2} \cosh ^{-2}\left(\frac{1}{2} \beta_{c}\left(q^{2}-\mu\right)\right) d q=\frac{h^{4} \beta_{c}^{2}}{2} \int_{\mathbb{R}^{d}} t(q)^{4} \frac{g_{1}\left(\beta_{c}\left(q^{2}-\mu\right)\right)}{q^{2}-\mu} d q
$$

according to our normalization (1.18). 
The second term on the right side of (4.7) can be rewritten as

$$
\begin{aligned}
& -h^{2(1-d)} \int_{\mathbb{R}^{d} \times \mathcal{C}} V\left(h^{-1}(x-y)\right)\left|\frac{1}{2}(\psi(x)+\psi(y)) \alpha_{0}\left(h^{-1}(x-y)\right)\right|^{2} d x d y \\
& =-h^{2-d} \sum_{p \in(2 \pi \mathbb{Z})^{d}}|\widehat{\psi}(p)|^{2} \int_{\mathbb{R}^{d}} V(x)\left|\alpha_{0}(x)\right|^{2} \cos ^{2}\left(\frac{h}{2} p \cdot x\right) d x \\
& =\frac{h^{2-d} \beta_{c}}{16} \sum_{p}|\widehat{\psi}(p)|^{2} \int_{\mathbb{R}^{d}} t(q) g_{0}\left(\beta_{c}\left(q^{2}-\mu\right)\right)(2 t(q)+t(q-h p)+t(q+h p)) d q .
\end{aligned}
$$

By writing

$$
\begin{aligned}
& 2 t(q)+t(q-h p)+t(q+h p) \\
& =4 t(q)+h^{2}\left[(p \cdot \nabla)^{2} t\right](q)+\frac{h^{4}}{6} \int_{-1}^{1}\left[(p \cdot \nabla)^{4} t\right](q+s h p)(1-|s|)^{3} d s
\end{aligned}
$$

we observe that (4.10) equals

$$
\begin{aligned}
& \frac{h^{2-d} \beta_{c}}{4}\|\psi\|_{2}^{2} \int_{\mathbb{R}^{d}} t(q)^{2} g_{0}\left(\beta_{c}\left(q^{2}-\mu\right)\right) d q \\
& +\frac{h^{4-d} \beta_{c}}{16} \sum_{i, j=1}^{d}\left\langle\partial_{i} \psi \mid \partial_{j} \psi\right\rangle \int_{\mathbb{R}^{d}} t(q)\left[\partial_{i} \partial_{j} t\right](q) g_{0}\left(\beta_{c}\left(q^{2}-\mu\right)\right) d q+O\left(h^{6-d}\right),
\end{aligned}
$$

where the error term is bounded by

$$
C h^{6-d}\|\psi\|_{H^{2}}^{2} \int_{\mathbb{R}^{d}}|V(x)|\left|\alpha_{0}(x)\right|^{2}|x|^{4} d x .
$$

The latter integral is shown to be finite in Proposition 1 in Appendix A.

If $V \leq 0$, the last term in (4.7) can be dropped for an upper bound, but we do not need to make this assumption. Since $V$ is relatively bounded with respect to the Laplacian, we can bound the term by an appropriate $H^{1}$-norm. Recall the definition of the $H^{1}$-norm of a periodic operator in (2.16). For general periodic operators $O$, we have the bound

$$
\begin{aligned}
& \left.\left|\int_{\mathcal{C} \times \mathbb{R}^{d}} V\left(h^{-1}(x-y)\right)\right| O(x, y)\right|^{2} d x d y \mid \\
& \leq\left\|\left(1-h^{2} \nabla^{2}\right)^{-1 / 2} V\left(h^{-1}(\cdot-y)\right)\left(1-h^{2} \nabla^{2}\right)^{-1 / 2}\right\|\|O\|_{H^{1}}^{2} \\
& =\left\|\left(1-\nabla^{2}\right)^{-1 / 2} V(\cdot)\left(1-\nabla^{2}\right)^{-1 / 2}\right\|\|O\|_{H^{1}}^{2} .
\end{aligned}
$$

The operator in question can be written as

$$
O=\alpha_{\Delta}-\frac{h}{2}\left(\psi(x) \widehat{\alpha}_{0}(-i h \nabla)+\widehat{\alpha}_{0}(-i h \nabla) \psi(x)\right) .
$$

Recall the definition of $\varphi$ in (2.17). It equals $\widehat{\alpha}_{0}$ for $\beta=\beta_{c}$. Hence

$$
\begin{aligned}
O= & \alpha_{\Delta}-\frac{h}{2}(\psi(x) \varphi(-i h \nabla)+\varphi(-i h \nabla) \psi(x)) \\
& +\frac{h}{4}(\psi(x) \eta(-i h \nabla)+\eta(-i h \nabla) \psi(x)),
\end{aligned}
$$

with

$$
\eta(q)=\left(\beta g_{0}\left(\beta\left(q^{2}-\mu\right)\right)-\beta_{c} g_{0}\left(\beta_{c}\left(q^{2}-\mu\right)\right)\right) t(q) .
$$


For the term in the first line of (4.16), we can apply Theorem 3 to bound its $H^{1}$ norm by $C h^{3-d / 2}\left(\|\psi\|_{H^{2}}+\|\psi\|_{H^{1}}^{3}\right)$. The $H^{1}$-norm of the term in the second line of (4.16) can be bounded by

$$
C h^{1-d / 2}\|\psi\|_{H^{1}}\left(\int_{\mathbb{R}^{d}}|\eta(q)|^{2}\left(1+q^{2}\right) d q\right)^{1 / 2} .
$$

It is easy to see that $|\eta(q)| \leq C\left(\beta-\beta_{c}\right)|t(q)| /\left(1+q^{2}\right)$; hence (4.18) is bounded by $C h^{3-d / 2}\|\psi\|_{H^{1}}$.

For $\psi$, we shall take a minimizer of the GL functional (1.7). Under our Assumption 2 on $W$ and $A$, it is easily seen to be in $H^{2}$. For this choice of $\psi$, we thus have

$$
F^{\mathrm{BCS}}\left(T_{c}\left(1-h^{2} D\right), \mu\right) \leq \mathcal{F}^{\mathrm{BCS}}\left(\Gamma_{\Delta}\right)-\mathcal{F}^{\mathrm{BCS}}\left(\Gamma_{0}\right) \leq h^{4-d}\left(E^{\mathrm{GL}}-B_{3}+C h\right)
$$

for small $h$. This completes the proof of the upper bound.

\section{Proof of Theorem 1; Lower bound, Part A}

Our proof of the lower bound on $F^{\mathrm{BCS}}(T, \mu)$ in Theorem 1 consists of two main parts. The goal of this first part is to show the following. Again let $\Gamma_{0}$ denote the normal state defined in (1.9), which is the minimizer of $\mathcal{F}^{\mathrm{BCS}}$ in the non-interacting case $V=0$. We claim that for any state $\Gamma$ satisfying $\mathcal{F}^{\mathrm{BCS}}(\Gamma) \leq \mathcal{F}^{\mathrm{BCS}}\left(\Gamma_{0}\right)$, we can decompose its off-diagonal part $\alpha$ as

$$
\alpha=\frac{h}{2}\left(\psi(x) \widehat{\alpha}_{0}(-i h \nabla)+\widehat{\alpha}_{0}(-i h \nabla) \psi(x)\right)+\xi
$$

for some periodic function $\psi$ with $H^{1}(\mathcal{C})$-norm bounded independently of $h$, and with $\|\xi\|_{H^{1}} \leq O\left(h^{2-d / 2}\right)$, where we use again the definition (2.16) for the $H^{1}$-norm of a periodic operator. This latter bound has to be compared with the $H^{1}$-norm of the first part of (5.1), which is $O\left(h^{1-d / 2}\right)$ (for fixed $\psi \neq 0$.)

The remainder of this section contains the proof of (5.1). It will be the crucial ingredient in the second part in the next section. The proof is divided into three steps.

5.1. Step 1. We claim that for any state $\Gamma$ of the form (1.2) satisfying $\mathcal{F}^{\mathrm{BCS}}(\Gamma) \leq$ $\mathcal{F}^{\mathrm{BCS}}\left(\Gamma_{0}\right)$, we have that

$$
\frac{4 T}{5} \operatorname{Tr}(\alpha \bar{\alpha})^{2}+\int_{\mathcal{C}}\left\langle\alpha(\cdot, y)\left|K_{T}^{A, W}+V\left(h^{-1}(\cdot-y)\right)\right| \alpha(\cdot, y)\right\rangle d y \leq 0 .
$$

Here, $K_{T}^{A, W}$ denotes the operator

$$
K_{T}^{A, W}=\frac{(-i h \nabla+h A(x))^{2}-\mu+h^{2} W(x)}{\tanh \left(\frac{\beta}{2}\left((-i h \nabla+h A(x))^{2}-\mu+h^{2} W(x)\right)\right)},
$$

with $\beta=1 / T$. In (5.2), it acts on the $x$-variable of $\alpha(x, y)$, and $\langle\cdot \mid \cdot\rangle$ denotes the standard inner product on $L^{2}\left(\mathbb{R}^{d}\right)$. Note that $K_{T}^{0,0}$ differs from the operator $K_{T}$ defined in (1.6) by a scaling by $h$. For $T=T_{c}$, the ground state of $K_{T_{c}}^{0,0}+V\left(h^{-1}(\cdot-\right.$ $y)$ ) equals $h^{-d / 2} \alpha_{0}\left(h^{-1}(x-y)\right)$ (up to an $h$-independent normalization).

Using that $(-i h \nabla+h A(x))^{2}-\mu+h^{2} W(x)=T \ln \left(\left(1-\gamma_{0}\right) / \gamma_{0}\right)$, we may write, for any state $\Gamma$,

$$
\mathcal{F}^{\mathrm{BCS}}(\Gamma)-\mathcal{F}^{\mathrm{BCS}}\left(\Gamma_{0}\right)=\frac{1}{2} T \mathcal{H}_{0}\left(\Gamma, \Gamma_{0}\right)+\int_{\mathcal{C} \times \mathbb{R}^{d}} V\left(h^{-1}(x-y)\right)|\alpha(x, y)|^{2} d x d y
$$


where $\mathcal{H}_{0}$ denotes the relative entropy

$$
\mathcal{H}_{0}\left(\Gamma, \Gamma_{0}\right)=\operatorname{Tr}_{0}\left[\Gamma\left(\ln \Gamma-\ln \Gamma_{0}\right)+(1-\Gamma)\left(\ln (1-\Gamma)-\ln \left(1-\Gamma_{0}\right)\right)\right] .
$$

We have the following lower bound.

Lemma 1. For any $0 \leq \Gamma \leq 1$ and any $\Gamma_{0}$ of the form $\Gamma_{0}=\left(1+e^{H}\right)^{-1}$ commuting with $P_{0}$ in (2.7),

$$
\mathcal{H}_{0}\left(\Gamma, \Gamma_{0}\right) \geq \operatorname{Tr}_{0}\left[\frac{H}{\tanh (H / 2)}\left(\Gamma-\Gamma_{0}\right)^{2}\right]+\frac{4}{3} \operatorname{Tr}\left[\Gamma(1-\Gamma)-\Gamma_{0}\left(1-\Gamma_{0}\right)\right]^{2} .
$$

A similar bound as (5.6), without the last positive term, was used in [11. Since the last term is the trace of a positive operator, $\operatorname{Tr}$ can be replaced by $\operatorname{Tr}_{0}$ there.

Proof. It is elementary (but tedious) to show that for real numbers $0<x, y<1$,

$$
x \ln \frac{x}{y}+(1-x) \ln \frac{1-x}{1-y} \geq \frac{\ln \frac{1-y}{y}}{1-2 y}(x-y)^{2}+\frac{4}{3}(x(1-x)-y(1-y))^{2} .
$$

The result then follows from Klein's inequality (see (3.4) and the subsequent remark).

Note that for $\Gamma_{0}$ given in (1.9), the corresponding $H$ in Lemma 1 equals $\beta H_{0}$, which is diagonal as an operator-valued $2 \times 2$ matrix. Hence also $H_{0} / \tanh \left(\beta H_{0} / 2\right)$ is diagonal. Its diagonal entries are $\beta K_{T}^{A, W}$ and $\beta \bar{K}_{T}^{A, W}$, respectively, where $K_{T}^{A, W}$ is given in (5.3) above. Hence

$$
\operatorname{Tr}_{0}\left[\frac{H_{0}}{\tanh \left(\frac{\beta}{2} H_{0}\right)}\left(\Gamma-\Gamma_{0}\right)^{2}\right]=2 \operatorname{Tr}\left[K_{T}^{A, W}\left(\gamma-\gamma_{0}\right)^{2}\right]+2 \operatorname{Tr}\left[K_{T}^{A, W} \alpha \bar{\alpha}\right]
$$

Since $x / \tanh (x / 2) \geq 2$, we can replace $K_{T}^{A, W}$ by $2 T$ for a lower bound. We shall use this in the first term on the right side of (5.8).

For the last term in (5.6), we use

$$
\operatorname{Tr}\left[\Gamma(1-\Gamma)-\Gamma_{0}\left(1-\Gamma_{0}\right)\right]^{2} \geq 2 \operatorname{Tr}\left[\gamma(1-\gamma)-\gamma_{0}\left(1-\gamma_{0}\right)-\alpha \bar{\alpha}\right]^{2} .
$$

We claim that

$$
2 \operatorname{Tr}\left(\gamma-\gamma_{0}\right)^{2}+\frac{4}{3} \operatorname{Tr}\left[\gamma(1-\gamma)-\gamma_{0}\left(1-\gamma_{0}\right)-\alpha \bar{\alpha}\right]^{2} \geq \frac{4}{5} \operatorname{Tr}(\alpha \bar{\alpha})^{2} .
$$

This follows easily from the triangle inequality

$$
\|\alpha \bar{\alpha}\|_{2} \leq\left\|\gamma(1-\gamma)-\gamma_{0}\left(1-\gamma_{0}\right)-\alpha \bar{\alpha}\right\|_{2}+\left\|\gamma(1-\gamma)-\gamma_{0}\left(1-\gamma_{0}\right)\right\|_{2}
$$

together with the fact that

$$
\left\|\gamma(1-\gamma)-\gamma_{0}\left(1-\gamma_{0}\right)\right\|_{2} \leq\left\|\gamma-\gamma_{0}\right\|_{2},
$$

which can be seen using Klein's inequality (3.4), for instance.

This completes the proof of (5.2). 
5.2. Step 2. Recall that $K_{T_{c}}^{0,0}+V\left(h^{-1}(\cdot)\right)$ is non-negative and has a non-degenerate isolated eigenvalue zero. Hence it will be convenient to replace $K_{T}^{A, W}$ by $K_{T_{c}}^{0,0}$ in (5.2). The following lemma quantifies the effect of such a replacement.

Lemma 2. For $T=T_{c}-O\left(h^{2}\right)$ and $h$ small enough,

$$
K_{T}^{A, W}+V\left(h^{-1}(\cdot-y)\right) \geq \frac{1}{8}\left(K_{T_{c}}^{0,0}+V\left(h^{-1}(\cdot-y)\right)\right)-C h^{2}
$$

for a constant $C>0$ depending only on $\|W\|_{\infty},\|A\|_{C^{1}}$ and $h^{-2}\left(T-T_{c}\right)$.

The proof shows that the prefactor $1 / 8$ can be replaced by any number less than one, at the expense of an increase in the constant $C$.

Proof. We start by noting that [1, (4.3.91)]

$$
\frac{x}{\tanh (x / 2)}=2+\frac{1}{2} \sum_{k=1}^{\infty} \frac{x^{2}}{x^{2} / 4+k^{2} \pi^{2}}=2+\sum_{k=1}^{\infty}\left(2-\frac{2 k^{2} \pi^{2}}{x^{2} / 4+k^{2} \pi^{2}}\right) .
$$

In particular, this function is operator monotone as a function of $x^{2}$. Let $\widetilde{p}=$ $-i h \nabla+h A(y)$ and $\widetilde{A}(x)=A(x)-A(y)$. Since the ground state of the operator $K_{T_{c}}^{0,0}+V\left(h^{-1}(\cdot-y)\right)$ is localized within a distance $O(h)$ of $y, \widetilde{A}(x)$ is, as far as the ground state is concerned, effectively a perturbation of order $h$ since $A$ is assumed to be Lipschitz continuous. Using Schwarz's inequality,

$$
\begin{aligned}
& {\left[(\widetilde{p}+h \widetilde{A}(x))^{2}-\mu+h^{2} W(x)\right]^{2}} \\
& \geq(1-\epsilon)\left[\widetilde{p}^{2}+h \widetilde{p} \cdot \widetilde{A}(x)+h \widetilde{A}(x) \cdot \widetilde{p}-\mu\right]^{2}-\frac{h^{4}}{\epsilon}\left\|W+\widetilde{A}^{2}\right\|_{\infty}^{2} \\
& \geq(1-\epsilon)^{2}\left[\widetilde{p}^{2}-\mu\right]^{2}-\frac{h^{4}}{\epsilon}\left\|W+\widetilde{A}^{2}\right\|_{\infty}^{2}-\frac{h^{2}}{\epsilon}[\widetilde{p} \cdot \widetilde{A}(x)+\widetilde{A}(x) \cdot \widetilde{p}]^{2} .
\end{aligned}
$$

We write the right side as $\beta^{-2}(R-Q)$, where

$$
Q=\frac{h^{2} \beta^{2}}{\epsilon}[\widetilde{p} \cdot \widetilde{A}(x)+\widetilde{A}(x) \cdot \widetilde{p}]^{2}
$$

and

$$
R=\beta^{2}(1-\epsilon)^{2}\left[\widetilde{p}^{2}-\mu\right]^{2}-\frac{h^{4} \beta^{2}}{\epsilon}\left\|W+\widetilde{A}^{2}\right\|_{\infty}^{2} .
$$

As long as $R-Q+4 \pi^{2}>0$, we can use the operator monotonicity of (5.14) to obtain a lower bound. This condition is certainly satisfied for small enough values of $\epsilon, h^{2} / \epsilon$ and $h^{4} / \epsilon$. Hence we can use the resolvent identity to conclude that

$$
\begin{aligned}
\beta K_{T}^{A, W} \geq 2 & +\frac{1}{2} \sum_{k=1}^{\infty} \frac{R}{k^{2} \pi^{2}+R / 4} \\
& -\frac{1}{2} \sum_{k=1}^{\infty} \frac{k^{2} \pi^{2}}{k^{2} \pi^{2}+R / 4} Q \frac{1}{k^{2} \pi^{2}+R / 4} \\
& -\frac{1}{8} \sum_{k=1}^{\infty} \frac{k^{2} \pi^{2}}{k^{2} \pi^{2}+R / 4} Q \frac{1}{k^{2} \pi^{2}+(R-Q) / 4} Q \frac{1}{k^{2} \pi^{2}+R / 4} .
\end{aligned}
$$


Since also $R-Q>Q-4 \pi^{2}$ for $\epsilon, h^{2} / \epsilon$ and $h^{4} / \epsilon$ small enough, we can bound

$$
Q \frac{1}{k^{2} \pi^{2}+(R-Q) / 4} Q \leq 4 Q
$$

for all $k \geq 1$. We thus obtain the lower bound

$$
K_{T}^{A, W} \geq \frac{1}{\beta}\left(2+\frac{1}{2} \sum_{k=1}^{\infty} \frac{R}{k^{2} \pi^{2}+R / 4}\right)-\frac{1}{\beta} \sum_{k=1}^{\infty} \frac{k^{2} \pi^{2}}{k^{2} \pi^{2}+R / 4} Q \frac{1}{k^{2} \pi^{2}+R / 4}
$$

We start with deriving a lower bound on the first term on the right side of (5.20), and defer the discussion of the second term to (5.28) et seq. For $h^{4} / \epsilon$ small enough, the first term on the right side of (5.20) is bounded from below by

$$
(1-\epsilon)^{2} \beta K_{T}^{A_{y}, 0}-\frac{h^{4}}{\epsilon} \beta^{2}\left\|W+\widetilde{A}^{2}\right\|_{\infty}^{2} \frac{\pi^{2}}{12} \frac{1}{\pi^{2}-h^{4} \epsilon^{-1} \beta^{2}\left\|W+\widetilde{A}^{2}\right\|_{\infty}^{2}},
$$

where we denote by $A_{y}$ the constant vector potential $A(y)$. Moreover, it is elementary to show that

$$
K_{T}^{A_{y}, 0} \geq K_{T_{c}}^{A_{y}, 0}-2\left(T_{c}-T\right)
$$

for $T \leq T_{c}$. We further have

$$
\begin{aligned}
(1-2 \epsilon) K_{T_{c}}^{A_{y}, 0}+V\left(h^{-1}(\cdot-y)\right)= & (1-3 \epsilon)\left(K_{T_{c}}^{A_{y}, 0}+V\left(h^{-1}(\cdot-y)\right)\right) \\
& +\epsilon\left(K_{T_{c}}^{A_{y}, 0}+3 V\left(h^{-1}(\cdot-y)\right)\right) \\
\geq & (1-3 \epsilon)\left(K_{T_{c}}^{A_{y}, 0}+V\left(h^{-1}(\cdot-y)\right)\right)-C \epsilon,
\end{aligned}
$$

where $-C$ is the ground state energy of $K_{T_{c}}^{A_{y}, 0}+3 V$, which is bounded by our assumptions on $V$. It is also independent of $A_{y}$ since $A_{y}$ may be replaced by zero by a unitary (gauge) transformation.

We now want to get rid of the constant vector potential $A(y)$ in $K_{T_{c}}^{A_{y}, 0}$ on the right side of (5.23). We claim that

$$
K_{T_{c}}^{A_{y}, 0}+V\left(h^{-1}(\cdot-y)\right) \geq \frac{1}{2}\left(K_{T_{c}}^{0,0}+V\left(h^{-1}(\cdot-y)\right)\right)-C h^{2} A(y)^{2} .
$$

This follows from the fact that

$$
K_{T_{c}}^{A_{y}, 0} \geq \frac{1}{2} K_{T_{c}}^{0,0}+\frac{1}{2} K_{T_{c}}^{2 A_{y}, 0}-C h^{2} A(y)^{2}
$$

(since the function $p \mapsto\left(p^{2}-\mu\right) / \tanh \left[\frac{\beta}{2}\left(p^{2}-\mu\right)\right]$ has a bounded Hessian) and

$$
K_{T_{c}}^{2 A_{y}, 0}+V\left(h^{-1}(\cdot-y)\right) \geq 0
$$

by the definition of $T_{c}$.

For $3 \epsilon \leq \frac{1}{2}$ and $T_{c}-T=O\left(h^{2}\right)$, we conclude that

$$
\begin{aligned}
K_{T}^{A, W}+V\left(h^{-1}(\cdot-y)\right) \geq & \frac{1}{4}\left(K_{T_{c}}^{0,0}+V\left(h^{-1}(\cdot-y)\right)\right) \\
& -C\left(h^{2}+\epsilon+h^{4} \epsilon^{-1}\right)-E,
\end{aligned}
$$

where $E$ denotes the last term in (5.20). Let $P$ denote the projection onto the ground state of $K_{T_{c}}^{0,0}+V\left(h^{-1}(\cdot-y)\right)$, given by the function $\alpha_{0}\left(h^{-1}(\cdot-y)\right)$. Also let $P^{c}=1-P$. Since $E$ is positive, it follows from Schwarz's inequality that

$$
E \leq 2 P E P+2 P^{c} E P^{c} .
$$


From the assumption that $A \in C^{1}$ it follows easily that $0 \leq Q \leq C h^{2} \epsilon^{-1}\left(1+\widetilde{p}^{2}\right)$. This immediately implies that $E \leq C h^{2} \epsilon^{-1}\left(1+\widetilde{p}^{2}\right) \leq C h^{2} \epsilon^{-1}\left(1-h^{2} \nabla^{2}\right)$ (since $A$ is bounded). We shall choose $\epsilon=O\left(h^{2}\right)$, with $h^{2} / \epsilon$ small enough to ensure that

$$
\frac{1}{8}\left(K_{T_{c}}^{0,0}+V\left(h^{-1}(\cdot-y)\right)\right)-2 P^{c} E P^{c} \geq 0 .
$$

That this can be done follows from the above bound on $E$ and the fact that $K_{T_{c}}^{0,0}+$ $V\left(h^{-1}(\cdot-y)\right) \geq \nu P^{c}\left(1-h^{2} \nabla^{2}\right) P^{c}$ for some $\nu>0$.

It remains to show that $P E P \leq O\left(h^{2}\right)$. Since $P$ has rank one, we can write

$$
P E P=C h^{-d}\left\langle\alpha_{0}\left(h^{-1}(\cdot-y)\right)|E| \alpha_{0}\left(h^{-1}(\cdot-y)\right)\right\rangle P
$$

for some $h$-independent constant $C$ determined by the normalization of $\alpha_{0}$. We have

$$
\begin{aligned}
Q & =\frac{h^{2} \beta^{2}}{\epsilon}(2 \widetilde{p} \cdot \widetilde{A}(x)+i h \operatorname{div} A(x))(2 \widetilde{A}(x) \cdot \widetilde{p}-i h \operatorname{div} A(x)) \\
& \leq \frac{h^{2} \beta^{2}}{\epsilon}\left(8 \widetilde{p} \cdot \widetilde{A}(x) \widetilde{A}(x) \cdot \widetilde{p}+2 h^{2}(\operatorname{div} A(x))^{2}\right) .
\end{aligned}
$$

Note that the last term is bounded by our assumption $A \in C^{1}$. From the Lipschitz continuity of $A$ it follows that $|\widetilde{A}(x)| \leq C|x-y|$, and hence

$$
\widetilde{p} \cdot \widetilde{A}(x) \widetilde{A}(x) \cdot \widetilde{p} \leq C^{2} \widetilde{p} \cdot|x-y|^{2} \widetilde{p} .
$$

We thus have $P E P \leq C h^{2} P \sum_{k} k^{2} \lambda_{k}$, where

$$
\begin{aligned}
\lambda_{k}= & \frac{h^{2} \beta}{\epsilon} \sum_{i=1}^{d} \int_{\mathbb{R}^{d}}\left|\nabla_{p} \frac{p_{i}+h A_{i}(y)}{k^{2} \pi^{2}+\frac{1}{4} \beta^{2}(1-\epsilon)^{2}\left[\left(p+h A_{y}\right)^{2}-\mu\right]^{2}-\delta} \widehat{\alpha}_{0}(p)\right|^{2} d p \\
& +\frac{h^{2} \beta}{\epsilon}\|\operatorname{div} A\|_{\infty}^{2} \frac{1}{\left(k^{2} \pi^{2}-\delta\right)^{2}},
\end{aligned}
$$

with $\delta=O\left(h^{2}\right)$ denoting $\delta=\frac{1}{4} h^{4} \beta \epsilon^{-1}\|W+\widetilde{A}\|_{\infty}^{2}$. For small enough $h$, we can thus bound

$$
\lambda_{k} \leq C \frac{h^{2}}{\epsilon k^{4}} \int_{\mathbb{R}^{d}}\left(1+|x|^{2}\right)\left(\left|\nabla \alpha_{0}(x)\right|^{2}+\left|\alpha_{0}(x)\right|^{2}\right) d x .
$$

The latter integral is finite, as proved in Proposition 2 in Appendix A Hence $\sum_{k} k^{2} \lambda_{k}$ is bounded, uniformly in $h$ for small $h$. This completes the proof.

5.3. Step 3. In combination with Lemma 2, we conclude from (5.2) that for $T=$ $T_{c}-O\left(h^{2}\right)$,

$$
\frac{4 T}{5} \operatorname{Tr}[\alpha \bar{\alpha}]^{2}+\frac{1}{8} \int_{\mathcal{C}}\left\langle\alpha(\cdot, y)\left|K_{T_{c}}^{0,0}+V\left(h^{-1}(\cdot-y)\right)\right| \alpha(\cdot, y)\right\rangle d y \leq C h^{2}\|\alpha\|_{2}^{2}
$$

for any state $\Gamma$ with $\mathcal{F}^{\mathrm{BCS}}(\Gamma) \leq \mathcal{F}^{\mathrm{BCS}}\left(\Gamma_{0}\right)$. We shall now show that this inequality implies (5.1).

Recall that the operator $K_{T_{c}}^{0,0}+V\left(h^{-1}(\cdot-y)\right)$ on $L^{2}\left(\mathbb{R}^{d}\right)$ has a unique ground state, proportional to $\alpha_{0}\left(h^{-1}(x-y)\right)$, with ground state energy zero, and a gap above, which we shall denote by $\kappa>0$. Normalize $\alpha_{0}$ as in (1.18), and let

$$
\psi(y)=(2 \pi)^{d / 2}\left(h \int_{\mathbb{R}^{d}}\left|\alpha_{0}(x)\right|^{2} d x\right)^{-1} \int_{\mathbb{R}^{d}} \alpha_{0}\left(h^{-1}(x-y)\right) \alpha(x, y) d x .
$$


Note that $\psi$ is a periodic function. Define the operator $\xi_{0}$ by

$$
\alpha(x, y)=\psi(y) \frac{h^{1-d}}{(2 \pi)^{d / 2}} \alpha_{0}\left(h^{-1}(x-y)\right)+\xi_{0}(x, y) .
$$

Then

$$
\begin{aligned}
& \int_{\mathcal{C}}\left\langle\alpha(\cdot, y)\left|K_{T_{c}}^{0,0}+V\left(h^{-1}(\cdot-y)\right)\right| \alpha(\cdot, y)\right\rangle d y \\
& =\int_{\mathcal{C}}\left\langle\xi_{0}(\cdot, y)\left|K_{T_{c}}^{0,0}+V\left(h^{-1}(\cdot-y)\right)\right| \xi_{0}(\cdot, y)\right\rangle d y \geq \kappa\left\|\xi_{0}\right\|_{2}^{2} .
\end{aligned}
$$

Together with (5.35), this yields $\left\|\xi_{0}\right\|_{2} \leq O(h)\|\alpha\|_{2}$. We can also symmetrize and write

$$
\alpha(x, y)=\frac{1}{2}(\psi(x)+\psi(y)) \frac{h^{1-d}}{(2 \pi)^{d / 2}} \alpha_{0}\left(h^{-1}(x-y)\right)+\xi(x, y),
$$

again with $\|\xi\|_{2} \leq O(h)\|\alpha\|_{2}$. In order to complete the proof of (5.1), we need to show that $\|\psi\|_{H^{1}}$ is bounded independently of $h$ and that the $H^{1}$-norm of $\xi$ is bounded by $O\left(h^{2-d / 2}\right)$.

An application of Schwarz's inequality yields

$$
\int_{\mathcal{C}}|\psi(x)|^{2} d x \leq(2 \pi)^{d} h^{d-2} \frac{\|\alpha\|_{2}^{2}}{\int_{\mathbb{R}^{d}}\left|\alpha_{0}(x)\right|^{2} d x} .
$$

Moreover,

$$
\|\alpha\|_{2}^{2} \leq \frac{h^{2-d}}{(2 \pi)^{d}} \int_{\mathcal{C}}|\psi(x)|^{2} d x \int_{\mathbb{R}^{d}}\left|\alpha_{0}(x)\right|^{2} d x+\left\|\xi_{0}\right\|_{2}^{2} .
$$

Since $\left\|\xi_{0}\right\|_{2} \leq O(h)\|\alpha\|_{2}$, we see that

$$
\|\alpha\|_{2}^{2} \leq\left(1+O\left(h^{2}\right)\right) \frac{h^{2-d}}{(2 \pi)^{d}} \int_{\mathcal{C}}|\psi(x)|^{2} d x \int_{\mathbb{R}^{d}}\left|\alpha_{0}(x)\right|^{2} d x .
$$

Again by using Schwarz's inequality,

$$
\int_{\mathcal{C}}|\nabla \psi(x)|^{2} d x \leq(2 \pi)^{d} h^{d-2} \frac{\int_{\mathbb{R}^{d} \times \mathcal{C}}\left|\left(\nabla_{x}+\nabla_{y}\right) \alpha(x, y)\right|^{2} d x d y}{\int\left|\alpha_{0}(x)\right|^{2} d x} .
$$

In order to bound the latter expression, we use the following lemma.

Lemma 3. For some constant $C>0$,

$$
\begin{aligned}
& h^{2} \int_{\mathbb{R}^{d} \times \mathcal{C}}\left|\left(\nabla_{x}+\nabla_{y}\right) \alpha(x, y)\right|^{2} d x d y \\
& \leq C \int_{\mathcal{C}}\left\langle\alpha(\cdot, y)\left|K_{T_{c}}^{0,0}+V\left(h^{-1}(\cdot-y)\right)\right| \alpha(\cdot, y)\right\rangle d y
\end{aligned}
$$

for all periodic and symmetric $\alpha$ (i.e., $\alpha(x, y)=\alpha(y, x))$.

Proof. By expanding $\alpha(x, y)$ in a Fourier series

$$
\alpha(x, y)=\sum_{p \in(2 \pi \mathbb{Z})^{d}} e^{i p \cdot(x+y) / 2} \widetilde{\alpha}_{p}(x-y)
$$

and using that $\widetilde{\alpha}_{p}(x)=\widetilde{\alpha}_{p}(-x)$ for all $p \in(2 \pi \mathbb{Z})^{d}$ we see that (5.44) is equivalent to

$$
K_{T_{c}}^{\frac{1}{2} p, 0}+K_{T_{c}}^{-\frac{1}{2} p, 0}+2 V(x / h) \geq \frac{2}{C} h^{2} p^{2}
$$


for all $p \in(2 \pi \mathbb{Z})^{d}$. This inequality holds for all $p \in \mathbb{R}^{d}$, in fact, for an appropriate choice of $C>0$, as we shall now show.

Since $K_{T_{c}}^{\frac{1}{2} p, 0} \geq C\left(1+h^{2}(-i \nabla+p / 2)^{2}\right)$, it suffices to consider the case of $h p$ small. Let $\kappa$ denote the gap in the spectrum of $K_{T_{c}}^{0,0}+V\left(h^{-1} \cdot\right)$ above zero, and $\varphi_{0}^{h}(x):=h^{-d / 2} \varphi_{0}(x / h)$ its normalized ground state. (Note that $\varphi_{0}$ is normalized to one, and hence equals a constant times $\alpha_{0}$.) Then

$$
\begin{aligned}
& K_{T_{c}}^{\frac{1}{2} p, 0}+K_{T_{c}}^{-\frac{1}{2} p, 0}+2 V(x / h) \\
& \geq \kappa\left[e^{i x \cdot p / 2}\left(1-\left|\varphi_{0}^{h}\right\rangle\left\langle\varphi_{0}^{h}\right|\right) e^{-i x \cdot p / 2}+e^{-i x \cdot p / 2}\left(1-\left|\varphi_{0}^{h}\right\rangle\left\langle\varphi_{0}^{h}\right|\right) e^{i x \cdot p / 2}\right] \\
& \geq \kappa\left[1-\left.\left|\int_{\mathbb{R}^{d}}\right| \varphi_{0}(x)\right|^{2} e^{-i h x \cdot p} d x \mid\right],
\end{aligned}
$$

where the last expression is simply the lowest eigenvalue of the operator on the previous line. Since $\varphi_{0}$ is reflection-symmetric,

$$
\int_{\mathbb{R}^{d}}\left|\varphi_{0}(x)\right|^{2} e^{-i h x \cdot p} d x=\int_{\mathbb{R}^{d}}\left|\varphi_{0}(x)\right|^{2} \cos (h x \cdot p) d x=1-O\left(h^{2} p^{2}\right)
$$

for small $h p$, since $\int_{\mathbb{R}^{d}}|x|^{2}\left|\varphi_{0}(x)\right|^{2} d x$ is finite by Proposition 2 ,

By combining (5.44) with (5.42), (5.43) and (5.35) we see that $\|\nabla \psi\|_{2}$ is bounded by a constant times $\|\psi\|_{2}$. To conclude the uniform upper bound on the $H^{1}$-norm of $\psi$, it thus suffices to give a bound on the $L^{2}$-norm. To do this, we have to utilize the first term on the left side of Eq. (5.35).

Recall that $\alpha$ can be decomposed as $\alpha=h \alpha_{0} \psi+\xi_{0}$, as in (5.37), where $\alpha_{0}$ is short for the operator $\widehat{\alpha}_{0}(-i h \nabla)$. The following lemma gives a lower bound on $\left(\operatorname{Tr}(\alpha \bar{\alpha})^{2}\right)^{1 / 4}$, the 4-norm of $\alpha$.

Lemma 4. For some $0<C<\infty$ we have

$$
\begin{aligned}
\|\alpha\|_{4} \geq & {\left[h^{4-d} \int_{\mathcal{C}}|\psi(x)|^{4} d x \int_{\mathbb{R}_{d}} \widehat{\alpha}_{0}(q)^{4} \frac{d q}{(2 \pi)^{d}}-C h^{5-d}\|\psi\|_{H^{1}(\mathcal{C})}^{4}\right]_{+}^{1 / 4} } \\
& -C h^{1-d / 4}\|\psi\|_{2}^{1 / 2}\left(1+C h^{1-d / 4}\|\psi\|_{4}\right)^{1 / 2}
\end{aligned}
$$

where $[\cdot]_{+}=\max \{0, \cdot\}$ denotes the positive part.

Proof. By the triangle inequality,

$$
\|\alpha\|_{4} \geq h\left\|\alpha_{0} \psi\right\|_{4}-\left\|\xi_{0}\right\|_{4} .
$$

We can bound the last term as $\left\|\xi_{0}\right\|_{4}^{2} \leq\left\|\xi_{0}\right\|_{\infty}\left\|\xi_{0}\right\|_{2}$. Recall that we have already shown that $\left\|\xi_{0}\right\|_{2} \leq O(h)\|\alpha\|_{2} \leq O\left(h^{2-d / 2}\right)\|\psi\|_{2}$. Note that we cannot bound the $\infty$-norm simply by the 2 -norm, since the norms $\|\cdot\|_{p}$ defined via the trace per unit volume in (3.1) are, in general, not monotone decreasing in $p$. We can, however, use that $\left\|\xi_{0}\right\|_{\infty} \leq\|\alpha\|_{\infty}+h\left\|\alpha_{0} \psi\right\|_{\infty}$ and that $\|\alpha\|_{\infty} \leq 1$ for any admissible state. Moreover, we claim that

$$
\left\|\alpha_{0} \psi\right\|_{\infty} \leq C_{\nu}\|\psi\|_{4} h^{-d / 4}\left(\int_{\mathbb{R}^{d}}\left|\alpha_{0}(x)(1+|x|)^{\nu}\right|^{4 / 3} d x\right)^{3 / 4}
$$

for $\nu>d$; that the latter integral is finite follows from the last statement in Proposition 2, which says that $\alpha_{0}(\cdot)(1+|\cdot|)^{\nu}$ is in $L^{2}\left(\mathbb{R}^{d}\right)$ for any $\nu>0$. 
Equation (5.51) can be obtained with the aid of Young's inequality [20, Thm. 4.2], as we now explain. With $\chi$ denoting the characteristic function of the unit cube, and $\chi_{j}(x)=\chi(x-j)$ for $j \in \mathbb{Z}^{d}$, we have, for any $f, g \in L^{2}\left(\mathbb{R}^{d}\right)$,

$$
\begin{aligned}
& \left|\left\langle f\left|\alpha_{0} \psi\right| g\right\rangle\right| \\
& \leq h^{-d} \sum_{j, k \in \mathbb{Z}^{d}}\left(1+\frac{[|j-k|-\sqrt{d}]_{+}}{h}\right)^{-\nu} \\
& \quad \times \int_{\mathbb{R}^{d}}\left|\chi_{j}(x) f(x) \alpha_{0}\left(\frac{x-y}{h}\right)\left(1+\frac{|x-y|}{h}\right)^{\nu} \psi(y) \chi_{k}(y) g(y)\right| d x d y \\
& \leq C h^{-d / 4} \sum_{j, k \in \mathbb{Z}^{d}}\left(1+\frac{[|j-k|-\sqrt{d}]_{+}}{h}\right)^{-\nu}\left\|\chi_{j} f\right\|_{2}\left\|\chi_{k} g\right\|_{2}\|\psi\|_{L^{4}(\mathcal{C})}\left\|\alpha_{0}(1+|\cdot|)^{\nu}\right\|_{4 / 3},
\end{aligned}
$$

where we used Young's inequality in the second step. We can further bound $\left\|\chi_{j} f\right\|_{2}\left\|\chi_{k} g\right\|_{2} \leq \lambda\left\|\chi_{j} f\right\|_{2}^{2}+\lambda^{-1}\left\|\chi_{k} g\right\|_{2}^{2}$ for $\lambda>0$. After doing the sum over $j$ and $k$ and optimizing over $\lambda$, we thus have

$$
\begin{aligned}
& \left|\left\langle f\left|\alpha_{0} \psi\right| g\right\rangle\right| \\
& \leq C h^{-d / 4}\|f\|_{2}\|g\|_{2} \sum_{j \in \mathbb{Z}^{d}}\left(1+\frac{[|j|-\sqrt{d}]_{+}}{h}\right)^{-\nu}\|\psi\|_{L^{4}(\mathcal{C})}\left\|\alpha_{0}(1+|\cdot|)^{\nu}\right\|_{4 / 3},
\end{aligned}
$$

which yields (5.51).

We have thus shown that

$$
\left\|\xi_{0}\right\|_{4}^{2} \leq C h^{2-d / 2}\|\psi\|_{2}\left(1+C h^{1-d / 4}\|\psi\|_{4}\right) .
$$

It remains to investigate the first term in (5.50). A short calculation shows that

$$
\left\|\alpha_{0} \psi\right\|_{4}^{4}=h^{-d} \sum_{p_{1}, p_{2}, p_{3}} \widehat{\psi}\left(p_{1}\right) \widehat{\psi}^{*}\left(p_{2}\right) \widehat{\psi}\left(p_{3}\right) \widehat{\psi}^{*}\left(-p_{1}-p_{2}-p_{3}\right) F\left(h p_{1}, h p_{2}, h p_{3}\right)
$$

where

$$
F\left(p_{1}, p_{2}, p_{3}\right)=\int_{\mathbb{R}^{3}} \widehat{\alpha}_{0}(q) \widehat{\alpha}_{0}\left(q+p_{1}\right) \widehat{\alpha}_{0}\left(q+p_{1}+p_{2}\right) \widehat{\alpha}_{0}\left(q+p_{1}+p_{2}+p_{3}\right) \frac{d q}{(2 \pi)^{d}}
$$

Note that $F$ is bounded. In fact,

$$
\left|F\left(p_{1}, p_{2}, p_{3}\right)\right| \leq(2 \pi)^{-d}\left\|\widehat{\alpha}_{0}\right\|_{4}^{4}
$$

To see that this is finite, one can use that $\left|\widehat{\alpha}_{0}(p)\right| \leq C|t(p)| /\left(1+p^{2}\right)$ (from the definition (1.17) ) and that $t \in L^{q}$ with $q$ as in Proposition 2. In the same way, one can show that $F$ has a bounded derivative, and hence

$$
\left|F\left(p_{1}, p_{2}, p_{3}\right)-F(0,0,0)\right| \leq C\left(\left|p_{1}\right|+\left|p_{2}\right|+\left|p_{3}\right|\right) .
$$


We are left with giving a bound on

$$
\begin{aligned}
& \sum_{p_{1}, p_{2}, p_{3}}\left|\widehat{\psi}\left(p_{1}\right) \widehat{\psi}^{*}\left(p_{2}\right) \widehat{\psi}\left(p_{3}\right) \widehat{\psi}^{*}\left(-p_{1}-p_{2}-p_{3}\right)\right|\left|p_{1}\right| \\
\leq & \left(\sum_{p}|\widehat{\psi}(p)|^{2}|p|^{2}\right)^{2}\left(\sum_{p}\left|\sum_{p_{2}, p_{3}}\right| \widehat{\psi}^{*}\left(p_{2}\right) \widehat{\psi}\left(p_{3}\right) \widehat{\psi}^{*}\left(-p-p_{2}-p_{3}\right)||^{2}\right)^{1 / 2} \\
= & \left(\int_{\mathcal{C}}|\nabla \psi(x)|^{2} d x\right)^{1 / 2}\left(\int_{\mathcal{C}}|\widetilde{\psi}(x)|^{6} d x\right)^{1 / 2},
\end{aligned}
$$

where $\widetilde{\psi}$ is the function whose Fourier transform equals $|\widehat{\psi}|$. For $d \leq 3$, we can use the Sobolev inequality

$$
\left(\int_{\mathcal{C}}|\widetilde{\psi}(x)|^{6} d x\right)^{1 / 3} \leq C\|\widetilde{\psi}\|_{H^{1}(\mathcal{C})}^{2}=C\|\psi\|_{H^{1}(\mathcal{C})}^{2} .
$$

Hence

$$
\left\|\alpha_{0} \psi\right\|_{4}^{4} \geq h^{-d} \int_{\mathcal{C}}|\psi(x)|^{4} d x \int_{\mathbb{R}^{d}} \widehat{\alpha}_{0}(q)^{4} \frac{d q}{(2 \pi)^{d}}-C h^{1-d}\|\psi\|_{H^{1}(\mathcal{C})}^{4},
$$

and this completes the proof.

We have already shown that $\|\nabla \psi\|_{2} \leq C\|\psi\|_{2}$, which also implies that $\|\psi\|_{4} \leq$ $C\|\psi\|_{2}$ via Sobolev's inequality for functions on the torus. If we also use that $\|\psi\|_{4} \geq\|\psi\|_{2}$ (since the norms of $\psi$ are defined via integration over the unit cube $\mathcal{C}$ ), we conclude from (5.49) that $\|\alpha\|_{4} \geq C h^{1-d / 4}\left(\|\psi\|_{2}-C\|\psi\|_{2}^{1 / 2}\right.$ ) for $h$ small enough. In combination with (5.35) and (5.42) this implies that $\|\psi\|_{2} \leq C$. This shows that the $H^{1}$-norm of $\psi$ is indeed uniformly bounded.

It follows that $\|\xi\|_{2} \leq O\left(h^{2-d / 2}\right)$. To conclude the proof of (5.1), we need to show that also $\|\xi\|_{H^{1}} \leq O\left(h^{2-d / 2}\right)$, i.e., $\|\nabla \xi\|_{2} \leq O\left(h^{1-d / 2}\right)$. We have

$$
\xi(x, y)=\xi_{0}(x, y)+\frac{1}{2}(\psi(x)-\psi(y)) \frac{h^{1-d}}{(2 \pi)^{d / 2}} \alpha_{0}\left(h^{-1}(x-y)\right) .
$$

From the definition (5.37) it follows easily that $\left\|\xi_{0}\right\|_{H^{1}} \leq O\left(h^{2-d / 2}\right)$, since

$$
\begin{aligned}
\left\|\xi_{0}\right\|_{H^{1}}^{2} & \leq C \int_{\mathcal{C}}\left\langle\xi_{0}(\cdot, y)\left|K_{T}^{0,0}+V\left(h^{-1}(\cdot-y)\right)+1\right| \xi_{0}(\cdot, y)\right\rangle d y \\
& \leq C\left(h^{2}\|\alpha\|_{2}^{2}+\left\|\xi_{0}\right\|_{2}^{2}\right) \leq O\left(h^{4-d}\right)
\end{aligned}
$$

using (5.35). Recall that the definition of the $H^{1}$-norm in (2.16) is not symmetric; hence this does not immediately imply a bound on the $H^{1}$-norm of $\xi$. However, we can estimate

$$
h^{2(1-d)} \int_{\mathcal{C}}|\nabla \psi(x)|^{2} \int_{\mathbb{R}^{d}}\left|\alpha_{0}\left(h^{-1}(x-y)\right)\right|^{2} d x d y \leq O\left(h^{2-d}\right) .
$$

Finally

$$
\begin{aligned}
& h^{-2 d} \int_{\mathcal{C} \times \mathbb{R}^{d}}|\psi(x)-\psi(y)|^{2}\left|\nabla \alpha_{0}\left(h^{-1}(x-y)\right)\right|^{2} d x d y \\
& =4 h^{-d} \sum_{p \in(2 \pi \mathbb{Z})^{d}}|\widehat{\psi}(p)|^{2} \int_{\mathbb{R}^{d}}\left|\nabla \alpha_{0}(x)\right|^{2} \sin ^{2}\left(\frac{1}{2} h p \cdot x\right) d x \leq O\left(h^{2-d}\right),
\end{aligned}
$$


since the $H^{1}$-norm of $\psi$ is bounded and $\int\left|\nabla \alpha_{0}\right|^{2}|x|^{2} d x$ is finite, as shown in Proposition 2 in Appendix A. This proves the claim.

\section{Proof of Theorem 1; Lower bound, Part B}

We now conclude the proof of the lower bound of Theorem 1 For convenience, we shall divide the proof into 2 steps.

6.1. Step 1. Let $\Gamma$ be a state with $\mathcal{F}^{\mathrm{BCS}}(\Gamma) \leq \mathcal{F}^{\mathrm{BCS}}\left(\Gamma_{0}\right)$. In the previous section, we have shown that the off-diagonal part $\alpha$ of $\Gamma$ can be decomposed in the form (5.1). Given $\psi$ defined in (5.1) and some $\epsilon>0$, define $\psi_{<}$via its Fourier transform

$$
\widehat{\psi}_{<}(p)=\widehat{\psi}(p) \theta\left(\epsilon h^{-1}-|p|\right),
$$

where $\theta(t)=1$ for $t \geq 1$, and 0 otherwise. The function $\psi_{<}$is thus smooth, and $\left\|\psi_{<}\right\|_{H^{2}} \leq C\left(1+\epsilon h^{-1}\right)$ since $\psi$ is bounded in $H^{1}$. We shall choose $h<\epsilon<1$.

Also let $\psi_{>}=\psi-\psi_{<}$. Since $\psi$ is bounded in $H^{1}$, the $L^{2}(\mathcal{C})$-norm of $\psi_{>}$is bounded by $O\left(h \epsilon^{-1}\right)$. We absorb the part $\frac{1}{2}\left(\psi_{>}(x)+\psi_{>}(y)\right) \alpha_{0}\left(h^{-1}(x-y)\right)$ into $\xi$ and write

$$
\alpha(x, y)=\frac{1}{2}\left(\psi_{<}(x)+\psi_{<}(y)\right) \frac{h^{1-d}}{(2 \pi)^{d / 2}} \alpha_{0}\left(h^{-1}(x-y)\right)+\sigma(x, y),
$$

where

$$
\sigma(x, y)=\xi(x, y)+\frac{1}{2}\left(\psi_{>}(x)+\psi_{>}(y)\right) \frac{h^{1-d}}{(2 \pi)^{d / 2}} \alpha_{0}\left(h^{-1}(x-y)\right) .
$$

In the previous section, we have shown that $\|\xi\|_{H^{1}} \leq O\left(h^{2-d / 2}\right)$. From the bound $\left\|\psi_{>}\right\|_{2} \leq O\left(h \epsilon^{-1}\right)$ it thus follows that $\|\sigma\|_{2} \leq O\left(h^{2-d / 2} \epsilon^{-1}\right)$. Note that we cannot conclude the same bound for the $H^{1}$-norm of $\sigma$, however.

As in (2.1), let $\Delta=-\frac{h}{2}\left(\psi_{<}(x) t(-i h \nabla)+t(-i h \nabla) \psi_{<}(x)\right)$. Its integral kernel is given in (4.2), with $\psi$ replaced by $\psi_{<}$. Let $H_{\Delta}$ be the corresponding Hamiltonian defined in (2.5). We can write

$$
\begin{aligned}
\mathcal{F}^{\mathrm{BCS}} & (\Gamma)-\mathcal{F}^{\mathrm{BCS}}\left(\Gamma_{0}\right) \\
= & -\frac{T}{2} \operatorname{Tr}_{0}\left[\ln \left(1+e^{-\beta H_{\Delta}}\right)-\ln \left(1+e^{-\beta H_{0}}\right)\right] \\
& -h^{2-2 d} \int_{\mathcal{C} \times \mathbb{R}^{d}} V\left(h^{-1}(x-y)\right) \frac{1}{4}\left|\psi_{<}(x)+\psi_{<}(y)\right|^{2}\left|\alpha_{0}\left(h^{-1}(x-y)\right)\right|^{2} \frac{d x d y}{(2 \pi)^{d}} \\
& +\frac{1}{2} T \mathcal{H}_{0}\left(\Gamma, \Gamma_{\Delta}\right)+\int_{\mathcal{C} \times \mathbb{R}^{d}} V\left(h^{-1}(x-y)\right)|\sigma(x, y)|^{2} d x d y,
\end{aligned}
$$

where $\mathcal{H}_{0}$ denotes the relative entropy defined in (5.5).

The terms in the first two lines on the right side of (6.4) have already been calculated. The first term is estimated in Theorem 2] and a bound on the second term was derived in Section 4 on the upper bound. The error in replacing $\beta$ with $\beta_{c}$ is as for the upper bound. Using the uniform upper bound on the $H^{1}$-norm of $\psi_{<}$, as well as $\left\|\psi_{<}\right\|_{H^{2}} \leq C \epsilon / h$, we obtain the lower bound

$$
\begin{aligned}
\mathcal{F}^{\mathrm{BCS}}(\Gamma)-\mathcal{F}^{\mathrm{BCS}}\left(\Gamma_{0}\right) \geq & h^{4-d}\left(\mathcal{E}^{\mathrm{GL}}\left(\psi_{<}\right)-B_{3}-C\left(h+\epsilon^{2}\right)\right) \\
& +\frac{1}{2} T \mathcal{H}_{0}\left(\Gamma, \Gamma_{\Delta}\right)+\int_{\mathcal{C} \times \mathbb{R}^{d}} V\left(h^{-1}(x-y)\right)|\sigma(x, y)|^{2} d x d y .
\end{aligned}
$$


It remains to show that the terms in the last line of (6.5) are negligible, i.e., of higher order than $h^{4-d}$, for an appropriate choice of $\epsilon \ll 1$. This will be accomplished in the next step.

6.2. Step 2. We again employ the method of Lemma 1 to get a lower bound on the relative entropy $\mathcal{H}_{0}\left(\Gamma, \Gamma_{\Delta}\right)$.

Lemma 5. For all admissible states $\Gamma$ with $\operatorname{Tr}\left(-\nabla^{2}+1\right) \gamma<\infty$,

$$
T \mathcal{H}_{0}\left(\Gamma, \Gamma_{\Delta}\right) \geq \operatorname{Tr}\left[\left(\Gamma-\Gamma_{\Delta}\right) \frac{H_{\Delta}}{\tanh \frac{1}{2} \beta H_{\Delta}}\left(\Gamma-\Gamma_{\Delta}\right)\right] .
$$

Proof. The inequality (6.6) would follow from (5.7) (dropping the last, positive term) and Klein's inequality (3.4) if we could evaluate the trace on the left side in the basis given by $H_{\Delta}$. Instead, we have to evaluate it in a basis of eigenfunctions of the projection $P_{0}$ defined in (2.7). In the following, we shall show that indeed we can evaluate the trace in (5.5) in the basis of $H_{\Delta}$ without changing its value. The proof of this fact is inspired by [12, Lemma 1].

The operators $\Gamma \ln \Gamma,(1-\Gamma) \ln (1-\Gamma), \Gamma_{\Delta} \ln \Gamma_{\Delta}$ and $\left(1-\Gamma_{\Delta}\right) \ln \left(1-\Gamma_{\Delta}\right)$ are locally trace class, since both states $\Gamma$ and $\Gamma_{\Delta}$ have finite kinetic energy and hence finite entropy (compare with Section 4). Therefore, it suffices to show that

$$
\operatorname{Tr}_{0} H_{\Delta}\left(\Gamma-\Gamma_{\Delta}\right)=\operatorname{Tr}_{\Delta} H_{\Delta}\left(\Gamma-\Gamma_{\Delta}\right),
$$

where $\operatorname{Tr}_{\Delta}$ denotes the trace per unit volume in the basis of $H_{\Delta}$. (More precisely, $H_{\Delta}$ can be written, via the Floquet decomposition, as a direct integral of operators on $L^{2}(\mathcal{C})$ each of which has discrete spectrum, and whose eigenfunctions serve as a basis to evaluate the trace $\operatorname{Tr}_{\Delta}$.)

Let $Q_{0}=1-P_{0}$, and let $P$ and $Q$ be arbitrary orthogonal projections with $P+Q=1$. For general operators $A$, we have

$$
P_{0} A P_{0}=P A P+\left(P_{0}-P\right) A P_{0}+P A\left(P_{0}-P\right)
$$

and hence

$$
P_{0} A P_{0}+Q_{0} A Q_{0}=P A P+Q A Q+E_{1}+E_{2}
$$

with

$$
E_{1}=\left(P_{0}-P\right) A\left(P_{0}-Q_{0}\right)
$$

and

$$
E_{2}=(P-Q) A\left(P_{0}-P\right) .
$$

We apply this to $A=H_{\Delta}\left(\Gamma-\Gamma_{\Delta}\right)$, and $P$ the projection on the positive spectral subspace of $H_{\Delta}$.

We claim that $\left|H_{\Delta}\right|^{1 / 2}\left(\Gamma-\Gamma_{\Delta}\right)$ is locally Hilbert-Schmidt. To see this, it is enough to show that $\left(\left|H_{0}\right|+1\right)^{1 / 2}\left(\Gamma-\Gamma_{\Delta}\right)$ is locally Hilbert-Schmidt, since $\Delta$ is bounded. This latter property follows from the finiteness of the kinetic energy of $\gamma$ and $\gamma_{\Delta}$, as well as the constraints on $\alpha$ and $\alpha_{\Delta}$ resulting from $0 \leq \Gamma \leq 1$ and $0 \leq \Gamma_{\Delta} \leq 1$, respectively. We also claim that $\left(P_{0}-P\right)\left|H_{\Delta}\right|^{1 / 2}$ is locally HilbertSchmidt. First note that $\left(1-\Gamma_{\Delta}-P\right)\left|H_{\Delta}\right|^{1 / 2}$ is a function of $H_{\Delta}$, and the estimate $\left|H_{\Delta}\right| \geq-c \nabla^{2}-C$ shows that it is locally Hilbert-Schmidt. Similarly one argues that also $\left(1-\Gamma_{0}-P_{0}\right)\left|H_{\Delta}\right|^{1 / 2}$ is locally Hilbert-Schmidt, using again the boundedness of $\Delta$. Hence the Hilbert-Schmidt property of $\left(P_{0}-P\right)\left|H_{\Delta}\right|^{1 / 2}$ follows from that of $\left(\Gamma_{0}-\Gamma_{\Delta}\right)\left|H_{\Delta}\right|^{1 / 2}$. 
We conclude that the operator $E_{1}$ is locally trace class. By cyclicity, its trace therefore equals that of

$$
\widetilde{E}_{1}=\left|H_{\Delta}\right|^{1 / 2}\left(\Gamma-\Gamma_{\Delta}\right)\left(P_{0}-Q_{0}\right)\left(P_{0}-P\right) H_{\Delta}^{1 / 2},
$$

where we denote $H_{\Delta}^{1 / 2}=H_{\Delta}\left|H_{\Delta}\right|^{-1 / 2}$. Since the left side of (6.9) (and hence also the right side) is locally trace class, we can evaluate the trace in the $H_{\Delta}$ basis. We have

$$
\operatorname{Tr}_{\Delta} E_{2}=\operatorname{Tr}_{\Delta}\left|H_{\Delta}\right|^{1 / 2}\left(\Gamma-\Gamma_{\Delta}\right)\left(P_{0}-P\right)(P-Q) H_{\Delta}^{1 / 2}=-\operatorname{Tr}_{\Delta} \widetilde{E}_{1}
$$

since

$$
\left(P_{0}-Q_{0}\right)\left(P_{0}-P\right)+\left(P_{0}-P\right)(P-Q)=0 .
$$

This completes the proof of (6.7). It then follows from Klein's inequality (see (3.4) and the subsequent remark) that

$$
\mathcal{H}_{0}\left(\Gamma, \Gamma_{\Delta}\right) \geq \operatorname{Tr}_{\Delta}\left[\frac{H_{\Delta}}{\tanh \frac{1}{2} \beta H_{\Delta}}\left(\Gamma-\Gamma_{\Delta}\right)^{2}\right] .
$$

The right side can, equivalently, be written as

$$
\operatorname{Tr}_{\Delta}\left[\left(\frac{H_{\Delta}}{\tanh \frac{1}{2} \beta H_{\Delta}}\right)^{1 / 2}\left(\Gamma-\Gamma_{\Delta}\right)^{2}\left(\frac{H_{\Delta}}{\tanh \frac{1}{2} \beta H_{\Delta}}\right)^{1 / 2}\right] .
$$

Since the operator in (6.16) is non-negative, $\operatorname{Tr}_{\Delta}$ can be replaced by the genuine trace per unit volume. Hence (6.16) equals the right side of (6.6).

As in the proof of Lemma 2, we shall use the fact that $x \mapsto \sqrt{x} / \tanh \sqrt{x}$ is an operator monotone function. An application of Schwarz's inequality yields

$$
H_{\Delta}^{2} \geq(1-\eta) H_{0}^{2}-\eta^{-1}\|\Delta\|_{\infty}^{2}
$$

for any $\eta>0$. To bound $H_{0}^{2}$ we proceed as in the proof of Lemma 2, specifically using (5.15) and (5.31), which states that $[-i \nabla A(x)-i A(x) \nabla]^{2} \leq C\left(1-\nabla^{2}\right)$. The choice $\epsilon=O(h)$ in (5.15) yields the lower bound

$$
H_{0}^{2} \geq(1-O(h))\left[-h^{2} \nabla^{2}-\mu\right]^{2} \otimes \mathbb{I}_{\mathbb{C}^{2}}-C h .
$$

The operator monotonicity thus implies that

$$
\begin{aligned}
K_{T}^{0,0} \otimes \mathbb{I}_{\mathbb{C}^{2}} & \leq \frac{(1-\eta-O(h))^{-1 / 2} \sqrt{H_{\Delta}^{2}+\eta^{-1}\|\Delta\|_{\infty}^{2}+C h}}{\tanh \left[\frac{1}{2} \beta(1-\eta-O(h))^{-1 / 2} \sqrt{H_{\Delta}^{2}+\eta^{-1}\|\Delta\|_{\infty}^{2}+C h}\right]} \\
& \leq(1-\eta-O(h))^{-1 / 2} \frac{\sqrt{\left.H_{\Delta}^{2}+\eta^{-1}\|\Delta\|_{\infty}^{2}+C h\right)}}{\tanh \left[\frac{1}{2} \beta \sqrt{H_{\Delta}^{2}+\eta^{-1}\|\Delta\|_{\infty}^{2}+C h}\right]} \\
& \leq(1-\eta-O(h))^{-1 / 2}\left(1+\frac{1}{4} \beta^{2}\left(\eta^{-1}\|\Delta\|_{\infty}^{2}+C h\right)\right) \frac{H_{\Delta}}{\tanh \frac{1}{2} \beta H_{\Delta}}
\end{aligned}
$$

for $0<\eta<1$. With the choice $\eta=O\left(\|\Delta\|_{\infty}\right)$ this gives

$$
\frac{H_{\Delta}}{\tanh \frac{1}{2} \beta H_{\Delta}} \geq\left(1-O\left(h+\|\Delta\|_{\infty}\right)\right) K_{T}^{0,0} \otimes \mathbb{I}_{\mathbb{C}^{2}} .
$$


In particular, we infer from (6.6) that

$$
\frac{T}{2} \mathcal{H}_{0}\left(\Gamma, \Gamma_{\Delta}\right) \geq\left(1-O\left(h+\|\Delta\|_{\infty}\right)\right) \operatorname{Tr}\left[K_{T}^{0,0}\left(\alpha-\alpha_{\Delta}\right)\left(\bar{\alpha}-\bar{\alpha}_{\Delta}\right)\right],
$$

where $\alpha_{\Delta}$ denotes again the upper off-diagonal entry of $\Gamma_{\Delta}$.

From the definition of $\Delta$, we see that

$$
\|\Delta\|_{\infty} \leq h\left\|\psi_{<}\right\|_{\infty}\|t\|_{\infty} .
$$

Moreover, since the Fourier transform of $\psi_{<}$is supported in the ball $|p| \leq \epsilon / h$,

$$
\begin{aligned}
\left\|\psi_{<}\right\|_{\infty} \leq \sum_{p}\left|\widehat{\psi}_{<}(p)\right| & \leq\left\|\psi_{<}\right\|_{H^{1}(\mathcal{C})}\left(\sum_{|p| \leq \epsilon h^{-1}} \frac{1}{1+p^{2}}\right)^{1 / 2} \\
& \leq C \times\left\{\begin{array}{cc}
1 & \text { for } d=1 \\
\sqrt{\ln (\epsilon / h)} & \text { for } d=2 \\
\sqrt{\epsilon / h} & \text { for } d=3 .
\end{array}\right.
\end{aligned}
$$

Recall the decomposition (6.2) of $\alpha$, and define $\phi$ by

$$
\alpha_{\Delta}=\frac{h}{2}\left(\psi_{<}(x) \widehat{\alpha}_{0}(-i h \nabla)+\widehat{\alpha}_{0}(-i h \nabla) \psi_{<}(x)\right)+\phi .
$$

We thus have

$$
\alpha-\alpha_{\Delta}=\sigma-\phi .
$$

Since $\left\|\psi_{<}\right\|_{H^{2}} \leq O(\epsilon / h)$, Theorem 3 implies that $\|\phi\|_{H^{1}} \leq O\left(\epsilon h^{2-d / 2}\right)$. From the positivity of $K_{T}^{0,0}$ we conclude that

$$
\operatorname{Tr}\left[K_{T}^{0,0}(\sigma-\phi)(\bar{\sigma}-\bar{\phi})\right] \geq \operatorname{Tr} K_{T}^{0,0} \sigma \bar{\sigma}-2 \operatorname{Re} \operatorname{Tr} K_{T}^{0,0} \bar{\sigma} \phi .
$$

The terms quadratic in $\sigma$ are thus

$$
(1-\delta) \operatorname{Tr} K_{T}^{0,0} \sigma \bar{\sigma}+\int_{\mathcal{C} \times \mathbb{R}^{d}} V\left(h^{-1}(x-y)\right)|\sigma(x, y)|^{2} d x d y
$$

with $\delta=O\left(h+\|\Delta\|_{\infty}\right)$. Pick some $\widetilde{\delta} \geq 0$ with $\delta+\widetilde{\delta} \leq 1 / 2$ and write

$$
(1-\delta) K_{T}^{0,0}+V=\widetilde{\delta} K_{T}^{0,0}+(1-2 \delta-2 \widetilde{\delta})\left(K_{T}^{0,0}+V\right)+(\delta+\widetilde{\delta})\left(K_{T}^{0,0}+2 V\right)
$$

$$
\geq \widetilde{\delta} K_{T}^{0,0}-2 D T_{c} h^{2}-C(\delta+\widetilde{\delta}),
$$

where we have used that $V$ is relatively form-bounded with respect to $K_{T}^{0,0}$ to bound the last term, and $K_{T}^{0,0} \geq K_{T_{c}}^{0,0}-2\left(T_{c}-T\right)=K_{T_{c}}^{0,0}-2 h^{2} D T_{c}$ to bound the second. Using also that $K_{T}^{0,0} \geq-h^{2} \nabla^{2}-\mu$, we thus conclude that

$$
\begin{aligned}
& (1-\delta) \operatorname{Tr} K_{T}^{0,0} \sigma \bar{\sigma}+\int_{\mathcal{C} \times \mathbb{R}^{d}} V\left(h^{-1}(x-y)\right)|\sigma(x, y)|^{2} d x d y \\
& \geq \widetilde{\delta}\left(\|\sigma\|_{H^{1}}^{2}-(C+\mu+1)\|\sigma\|_{2}^{2}\right)-\left(C \delta+2 D T_{c} h^{2}\right)\|\sigma\|_{2}^{2} .
\end{aligned}
$$

Recall that $\|\sigma\|_{2} \leq O\left(h^{2-d / 2} / \epsilon\right)$. We shall choose $\widetilde{\delta}=0$ if the first parenthesis on the right side of (6.29) is less than $\frac{1}{2}\|\sigma\|_{H^{1}}^{2}$ (and, in particular, if it is negative), while $\widetilde{\delta}=O(1)$ in the opposite case, i.e., when $\|\sigma\|_{H_{1}}^{2} \geq 2(C+\mu+1)\|\sigma\|_{2}^{2}$. In the latter case we shall have the positive term $\widetilde{\delta}\|\sigma\|_{H^{1}}^{2} / 2$ at our disposal, which will be used in (6.32) below. 
We are left with estimating the last term in (6.26). It can be bounded by the product of the $H^{1}$-norms of $\sigma$ and $\phi$. This turns out not to be good enough, however. Recall from (6.3) that $\sigma$ is a sum of two terms, $\xi$ and $\sigma-\xi$, where the latter is proportional to $\psi_{>}$, and $\|\xi\|_{H^{1}} \leq O\left(h^{2-d / 2}\right)$. Moreover, as the proof of Theorem 3 in Section 8 shows, $\phi$ is the sum of two terms, $\eta_{1}$ and $\phi-\eta_{1}$, with $\eta_{1}$ defined in (8.4) (with $\psi$ replaced by $\psi_{<}$) and $\left\|\phi-\eta_{1}\right\|_{H^{1}} \leq O\left(h^{3-d / 2}\right)$. Now

$$
\operatorname{Tr} K_{T}^{0,0}(\bar{\sigma}-\bar{\xi}) \eta_{1}=0
$$

as can be seen by writing out the trace in momentum space and using that $\widehat{\psi}_{<}$and $\widehat{\psi}_{>}$have disjoint support. Hence

$$
\begin{aligned}
\operatorname{Re} \operatorname{Tr} K_{T}^{0,0} \bar{\sigma} \phi & \leq C\left(\|\xi\|_{H^{1}}\|\phi\|_{H^{1}}+\|\sigma\|_{H^{1}}\left\|\phi-\eta_{1}\right\|_{H^{1}}\right) \\
& \leq O\left(\epsilon h^{4-d}\right)+O\left(h^{3-d / 2}\right)\|\sigma\|_{H^{1}} .
\end{aligned}
$$

In the case $\|\sigma\|_{H^{1}} \leq C\|\sigma\|_{2}$ (corresponding to $\widetilde{\delta}=0$ above) we can further bound $\|\sigma\|_{H^{1}} \leq O\left(h^{2-d / 2} / \epsilon\right)$. In the opposite case, where $\widetilde{\delta}=O(1)$, we can use the positive term $\widetilde{\delta}\|\sigma\|_{H^{1}}^{2} / 2$ from before and bound

$$
\frac{\widetilde{\delta}}{2}\|\sigma\|_{H^{1}}^{2}-O\left(h^{3-d / 2}\right)\|\sigma\|_{H^{1}} \geq-O\left(h^{6-d}\right),
$$

which thus leads to an even better bound.

In combination with (6.5) these bounds show that

$$
h^{d-4}\left(\mathcal{F}^{\mathrm{BCS}}(\Gamma)-\mathcal{F}^{\mathrm{BCS}}\left(\Gamma_{0}\right)\right) \geq \mathcal{E}^{\mathrm{GL}}\left(\psi_{<}\right)-B_{3}-C e,
$$

where

$$
e=h+\epsilon^{2}+\epsilon+\frac{h}{\epsilon}+\frac{h}{\epsilon^{2}} \times\left\{\begin{array}{cl}
1 & \text { for } d=1 \\
\sqrt{\ln (\epsilon / h)} & \text { for } d=2 \\
\sqrt{\epsilon / h} & \text { for } d=3 .
\end{array}\right.
$$

The choice $\epsilon=h^{1 / 3}$ for $d=1, \epsilon=h^{1 / 3}[\ln (1 / h)]^{1 / 6}$ for $d=2$ and $\epsilon=h^{1 / 5}$ for $d=3$, respectively, leads to

$$
e \leq C \times\left\{\begin{array}{cc}
h^{1 / 3} & \text { for } d=1 \\
h^{1 / 3}[\ln (1 / h)]^{1 / 6} & \text { for } d=2 \\
h^{1 / 5} & \text { for } d=3
\end{array}\right.
$$

This completes the lower bound to the BCS energy. The statement (1.15) about the minimizer follows immediately from (6.33) and (6.2).

\section{Proof of Theorem 2}

For simplicity, we prove Theorem 2 only in the case $d=3$. The cases $d=1$ and $d=2$ are very similar and are left to the reader.

Note that the function $f$ in (2.8) is real analytic and has a bounded derivative. The second and higher derivatives decay exponentially. In particular, the following lemma applies. It will be used repeatedly in the proof of Theorem 2 .

For a general smooth function $f: \mathbb{R} \rightarrow \mathbb{R}$, let $\left[a_{1}, \ldots, a_{N}\right]_{f}$ denote the divided differences [3], defined recursively via

$$
[a]_{f}=f(a), \quad\left[a_{1}, a_{2}\right]_{f}=\frac{f\left(a_{1}\right)-f\left(a_{2}\right)}{a_{1}-a_{2}}
$$


and

$$
\left[a_{1}, a_{2}, \ldots, a_{N}\right]_{f}=\frac{\left[a_{1}, \ldots, a_{N-1}\right]_{f}-\left[a_{2}, \ldots, a_{N}\right]_{f}}{a_{1}-a_{N}}
$$

in case $a_{1} \neq a_{N}$. The extension to coinciding arguments is simply by continuity. In the case of distinct arguments,

$$
\left[a_{1}, a_{2}, \ldots, a_{N}\right]_{f}=\sum_{j=1}^{N} f\left(a_{j}\right) \prod_{i, i \neq j}\left(a_{j}-a_{i}\right)^{-1} .
$$

In case $f$ is analytic in a neighborhood of the real axis, we have

$$
\left[a_{1}, \ldots, a_{N}\right]_{f}=\frac{1}{2 \pi i} \int_{\Gamma} f(z) \prod_{k=1}^{N} \frac{1}{z-a_{k}} d z,
$$

with $\Gamma$ a contour enclosing all the $a_{i}$.

Lemma 6. Let $f$ be a smooth function on $\mathbb{R}$ satisfying $\left|f^{(n)}(x)\right| \leq C(1+|x|)^{1-n}$ for $1 \leq n \leq N-1$. Then, for $N \geq 3$, there is a finite constant $C_{N}>0$ such that

$$
\left[a_{1}, \ldots, a_{N}\right]_{f} \leq \frac{C_{N}}{1+\max _{i}\left\{\left|a_{i}\right|\right\}} \quad \text { for all } a_{i} \in \mathbb{R} .
$$

Moreover, for all $1 \leq n<N$ there is a finite constant $C_{N}^{\prime}>0$ such that if $a_{i} \leq$ $-\lambda \leq 0$ for all $1 \leq i \leq n$ and $a_{i} \geq \lambda \geq 0$ for all $n+1 \leq i \leq N$, then

$$
\left[a_{1}, \ldots, a_{N}\right]_{f} \leq \frac{C_{N}^{\prime}}{(1+\lambda)^{N-2}} .
$$

Proof. We first prove (7.6). Using Feynman's formula

$$
\begin{aligned}
{\left[a_{1}, \ldots, a_{N}\right]_{f} } & =(N-1) ! \int_{[0,1]^{N}}\left[\sum_{i=1}^{N} c_{i} a_{i}, \ldots, \sum_{i=1}^{N} c_{i} a_{i}\right]_{f} \delta\left(1-\sum_{i=1}^{N} c_{i}\right) \prod_{k=1}^{N} d c_{k} \\
(7.7) \quad & =\int_{[0,1]^{N}} f^{(N-1)}\left(\sum_{i=1}^{N} c_{i} a_{i}\right) \delta\left(1-\sum_{i=1}^{N} c_{i}\right) \prod_{k=1}^{N} d c_{k}
\end{aligned}
$$

we see that $\left[a_{1}, \ldots, a_{N}\right]$ is uniformly bounded for $N \geq 2$. Hence it suffices to consider the case $\lambda \geq 1$. Similarly, a simple change of variables shows that

$$
\begin{aligned}
& {\left[a_{1}, \ldots, a_{N}\right]_{f}} \\
& =\int_{0}^{1} \int_{[0,1]^{N}} f^{(N-1)}\left(\gamma \sum_{i=1}^{n} c_{i} a_{i}+(1-\gamma) \sum_{i=n+1}^{N} c_{i} a_{i}\right) \\
& \quad \times \gamma^{n-1}(1-\gamma)^{N-n-1} \delta\left(1-\sum_{i=1}^{n} c_{i}\right) \delta\left(1-\sum_{i=n+1}^{N} c_{i}\right) \prod_{k=1}^{N} d c_{k} d \gamma
\end{aligned}
$$

and hence it is sufficient to consider the case $a_{1}=\cdots=a_{n}=a$ and $a_{n+1}=\cdots=$ $a_{N}=b$. In this special case, we have

$$
[a, \ldots, a, b, \ldots, b]_{f}=\frac{1}{(n-1) !(m-1) !}\left(\frac{\partial}{\partial a}\right)^{n-1}\left(\frac{\partial}{\partial b}\right)^{m-1} \frac{f(a)-f(b)}{a-b},
$$

where $m=N-n$. Note that $a \leq-\lambda \leq-1$ and $b \geq \lambda \geq 1$. The result now follows easily from our assumptions on $f$.

Let $\kappa=\max _{i}\left|a_{i}\right|$. To prove (7.5), we may again assume that $\kappa \geq 1$. If $\max _{i} a_{i}-$ $\min _{i} a_{i} \leq \kappa / 2$, then either $a_{i} \geq \kappa / 2$ for all $i$ or $a_{i} \leq-\kappa / 2$ for all $i$, and the result 
follows from (7.7). If, on the other hand, $\max _{i} a_{i}-\min _{i} a_{i} \geq \kappa / 2$, the result follows immediately from the definition (7.2), with $\min _{i} a_{i}$ in place of $a_{1}$ and $\max _{i} a_{i}$ in place of $a_{N}$, using the boundedness of the numerator.

What the lemma says is that $\left[a_{1}, a_{2}, \ldots, a_{N}\right]_{f}$ decays at least as fast as the inverse of its largest argument. If all the arguments get large, but in opposite directions, then the decay is at least as fast as the $(N-2)$ th power of the inverse.

Now let $f$ denote the function defined in (2.8). It is analytic in the strip $|\operatorname{Im} z|<$ $\pi$. This leads to the following contour integral representation.

Lemma 7. For $R>0$, let $\Gamma_{R}$ be the contour $\left\{r-i \frac{\pi}{2 \beta}, r \in[-R, R]\right\} \cup\left\{-r+i \frac{\pi}{2 \beta}, r \in\right.$ $[-R, R]\}$. Then

$$
f\left(\beta H_{\Delta}\right)-f\left(\beta H_{0}\right)=\lim _{R \rightarrow \infty} \frac{1}{2 \pi i} \int_{\Gamma_{R}} f(\beta z)\left(\frac{1}{z-H_{\Delta}}-\frac{1}{z-H_{0}}\right) d z,
$$

where the limit holds in the weak sense, i.e., for expectation values with functions in $C_{0}^{\infty}\left(\mathbb{R}^{d}\right) \oplus C_{0}^{\infty}\left(\mathbb{R}^{d}\right)$.

Since $f$ is unbounded, it is important to take the difference of the two operators $f\left(\beta H_{\Delta}\right)$ and $f\left(\beta H_{0}\right)$. The representation (7.10) would not hold for the individual terms alone. We shall write, for simplicity,

$$
f\left(\beta H_{\Delta}\right)-f\left(\beta H_{0}\right)=\frac{1}{2 \pi i} \int_{\Gamma} f(\beta z)\left(\frac{1}{z-H_{\Delta}}-\frac{1}{z-H_{0}}\right) d z
$$

where $\Gamma$ is the contour $z=r \pm i \frac{\pi}{2 \beta}, r \in \mathbb{R}$. This equality has to be understood as the weak limit (7.10).

Proof. For fixed $\lambda \in \mathbb{R}$, we have

$$
\lim _{R \rightarrow \infty} \frac{1}{2 \pi i} \int_{\Gamma_{R}} f(\beta z) \frac{1}{z-\lambda} d z=f(\beta \lambda)-\frac{1}{2}
$$

and the limit is uniform on bounded sets in $\lambda$. For functions $\phi$ in the range of $\theta\left(\lambda-\left|H_{\Delta}\right|\right)$ for some $\lambda>0$, this implies that

$$
\lim _{R \rightarrow \infty} \frac{1}{2 \pi i} \int_{\Gamma_{R}} f(\beta z)\left\langle\phi\left|\left(z-H_{\Delta}\right)^{-1}\right| \phi\right\rangle d z=\left\langle\phi\left|f\left(\beta H_{\Delta}\right)-\frac{1}{2}\right| \phi\right\rangle .
$$

Since the operators $\int_{\Gamma_{R}} f(\beta z)\left(z-H_{\Delta}\right)^{-1} d z$ are bounded, in absolute value, by $C\left(1+\left|H_{\Delta}\right|\right)$, uniformly in $R$, the limit extends to all functions $\phi$ in the form domain of $1+\left|H_{\Delta}\right|$. The same argument applies with $H_{0}$ in place of $H_{\Delta}$. The form domains of $1+\left|H_{\Delta}\right|$ and $1+\left|H_{0}\right|$ are equal since $\Delta$ is bounded, and contain $C_{0}^{\infty}\left(\mathbb{R}^{d}\right) \oplus C_{0}^{\infty}\left(\mathbb{R}^{d}\right)$. This completes the proof.

The diagonal entries of the $2 \times 2$ matrix-valued operator $f\left(\beta H_{\Delta}\right)-f\left(\beta H_{0}\right)$ are complex conjugates of each other. This follows from the unitary equivalence (4.3) and the fact that $f(-z)=f(z)-z$. The unitary $U$ in (4.3) interchanges the diagonal entries; hence the upper left entry of $f\left(\beta H_{\Delta}\right)-f\left(\beta H_{0}\right)$ equals the lower right entry of

$$
\begin{aligned}
U f\left(\beta H_{\Delta}\right) U^{\dagger}-U f\left(\beta H_{0}\right) U^{\dagger} & =f\left(-\beta \bar{H}_{\Delta}\right)-f\left(-\beta \bar{H}_{0}\right) \\
& =f\left(\beta \bar{H}_{\Delta}\right)-f\left(\beta \bar{H}_{0}\right)-\beta\left(\bar{H}_{\Delta}-\bar{H}_{0}\right) \\
& =\overline{f\left(\beta H_{\Delta}\right)}-\overline{f\left(\beta H_{0}\right)}-\beta\left(\bar{H}_{\Delta}-\bar{H}_{0}\right) .
\end{aligned}
$$


Since the diagonal entries of $H_{\Delta}-H_{0}$ are zero, this proves the claim. In particular, the diagonal entries of $f\left(\beta H_{\Delta}\right)-f\left(\beta H_{0}\right)$ have the same trace, and hence it suffices to study the upper left diagonal entry, which we denote by $[\cdot]_{11}$.

Let $k$ denote the operator

$$
k=(-i h \nabla+h A(x))^{2}-\mu+h^{2} W(x) .
$$

The resolvent identity and the fact that

$$
\delta:=H_{\Delta}-H_{0}=\left(\begin{array}{cc}
0 & \Delta \\
\bar{\Delta} & 0
\end{array}\right)
$$

is off-diagonal (as an operator-valued $2 \times 2$ matrix) imply that

$$
\left[\frac{1}{z-H_{\Delta}}-\frac{1}{z-H_{0}}\right]_{11}=I_{1}+I_{2}+I_{3}
$$

where

$$
\begin{gathered}
I_{1}=\frac{1}{z-k} \Delta \frac{1}{z+\bar{k}} \Delta^{\dagger} \frac{1}{z-k}, \\
I_{2}=\frac{1}{z-k} \Delta \frac{1}{z+\bar{k}} \Delta^{\dagger} \frac{1}{z-k} \Delta \frac{1}{z+\bar{k}} \Delta^{\dagger} \frac{1}{z-k},
\end{gathered}
$$

and

$$
I_{3}=\left[\frac{1}{z-H_{\Delta}}\right]_{11} \Delta \frac{1}{z+\bar{k}} \Delta^{\dagger} \frac{1}{z-k} \Delta \frac{1}{z+\bar{k}} \Delta^{\dagger} \frac{1}{z-k} \Delta \frac{1}{z+\bar{k}} \Delta^{\dagger} \frac{1}{z-k} .
$$

We shall first give a bound on the contribution of $I_{3}$ to the integral in (7.11).

\section{Lemma 8.}

$$
\left\|\int_{\Gamma} f(\beta z) I_{3} d z\right\|_{1} \leq C h^{6-d}\|\psi\|_{H^{1}(\mathcal{C})}^{6}\|t\|_{6}^{6}
$$

Proof. By the triangle inequality

$$
\left\|\int_{\Gamma} f(\beta z) I_{3} d z\right\|_{1} \leq \int_{\Gamma}|f(\beta z)|\left\|I_{3}\right\|_{1}|d z| .
$$

Using Hölder's inequality (3.9) and $\left|z-H_{\Delta}\right| \geq \pi /(2 \beta)$ for $z \in \Gamma$, we obtain the bound

$$
\left\|I_{3}\right\|_{1} \leq \frac{2 \beta}{\pi}\|\Delta\|_{6}^{6}\left\|(z-k)^{-1}\right\|_{\infty}^{3}\left\|(z+\bar{k})^{-1}\right\|_{\infty}^{3} .
$$

Moreover, since $k \geq-\mu-h^{2}\|W\|_{\infty}$,

$$
\left\|(z-k)^{-1}\right\|_{\infty} \leq C \times\left\{\begin{array}{cl}
1 & \text { for } r \gg 1 \\
O\left(|r|^{-1}\right) & \text { for } r \ll-1
\end{array}\right.
$$

for $z=r \pm i \pi /(2 \beta)$, and hence

$$
\left\|I_{3}\right\|_{1} \leq \frac{C}{1+|z|^{3}}\|\Delta\|_{6}^{6} .
$$

It is important to get a decay faster than $|z|^{-2}$ since we are integrating against the function $\left|\ln \left(1+e^{-\beta z}\right)\right|$ which grows linearly as $z \rightarrow-\infty$. We conclude that

$$
\int_{\Gamma}|f(\beta z)|\left\|I_{3}\right\|_{1}|d z| \leq C\|\Delta\|_{6}^{6} .
$$


We can further bound $\|\Delta\|_{6} \leq(2 \pi)^{-d / 6}\|\psi\|_{6} h^{1-d / 6}\|t\|_{6}$, according to the triangle inequality and (3.11). Finally, $\|\psi\|_{6} \leq C\|\psi\|_{H^{1}(\mathcal{C})}$ for $d \leq 3$, by Sobolev's inequality for functions on the torus. This completes the proof.

We continue with the bound on $I_{2}$. Recall the definition of the divided differences (7.1) - (7.2).

Lemma 9. The operator $\int_{\Gamma} f(\beta z) I_{2} d z$ is locally trace class, and

$$
\begin{aligned}
& \mid \frac{1}{2 \pi i} \operatorname{Tr} \int_{\Gamma} f(\beta z) I_{2} d z- \\
& h^{4-d} \sum_{p_{1}, p_{2}, p_{3} \in(2 \pi \mathbb{Z})^{d}} \widehat{\psi}\left(p_{1}\right) \widehat{\psi}^{*}\left(p_{2}\right) \widehat{\psi}\left(p_{3}\right) \widehat{\psi}^{*}\left(-p_{1}-p_{2}-p_{3}\right) F\left(h p_{1}, h p_{2}, h p_{3}\right) \mid \\
&(7.27) \quad \leq C h^{5-d}\|t\|_{6}^{4}\|\psi\|_{H^{1}(\mathcal{C})}^{4},
\end{aligned}
$$

where the function $F$ is given by

$$
F\left(p_{1}, p_{2}, p_{3}\right)
$$

$$
=\beta^{4} \int_{\mathbb{R}^{d}} \prod_{i=1}^{4} \frac{t\left(q+\sum_{j=1}^{i-1} p_{i}\right)+t\left(q+\sum_{j=1}^{i} p_{j}\right)}{2}\left[a_{3}, a_{3}, a_{1},-a_{2},-a_{0}\right]_{f} \frac{d q}{(2 \pi)^{d}}
$$

and where we introduced the notation $a_{i}=\beta\left(\left(q+\sum_{j=1}^{i} p_{i}\right)^{2}-\mu\right)$ and $p_{4}:=-p_{1}-$ $p_{2}-p_{3}$.

One can actually show that (17.27) holds with $h^{6-d}$ in place of $h^{5-d}$ in the error term. This requires substantially more effort, however, and hence we omit the proof for the sake of brevity.

Proof. The resolvent identity reads

$$
\frac{1}{z-k}=\frac{1}{z-k_{0}}+\frac{1}{z-k_{0}}\left(k-k_{0}\right) \frac{1}{z-k},
$$

where

$$
k_{0}=-h^{2} \nabla^{2}-\mu
$$

and hence

$$
k-k_{0}=h^{2} W(x)+h^{2} A(x)^{2}-i h^{2} \nabla \cdot A(x)-i h^{2} A(x) \cdot \nabla .
$$

We apply this to the first factor in $I_{2}$ in (7.19). Using again the Hölder inequality (3.9), we can bound

$$
\begin{aligned}
& \left\|\left(\frac{1}{z-k}-\frac{1}{z-k_{0}}\right) \Delta \frac{1}{z+\bar{k}} \Delta^{\dagger} \frac{1}{z-k} \Delta \frac{1}{z+\bar{k}} \Delta^{\dagger} \frac{1}{z-k}\right\|_{1} \\
& \leq\left\|\frac{1}{z-k_{0}}\left(k-k_{0}\right)\right\|_{\infty}\|\Delta\|_{6}^{4}\left\|(z-k)^{-1}\right\|_{\infty}^{3}\left\|(z+\bar{k})^{-1}\right\|_{6}^{2} .
\end{aligned}
$$

Using our assumptions on $A$ and $W$, it is easy to see that, for $z=r \pm i \pi /(2 \beta)$,

$$
\left\|\frac{1}{z-k_{0}}\left(-i h^{2} \nabla A(x)-i h^{2} A(x) \nabla\right)\right\|_{\infty} \leq C h \times \begin{cases}\sqrt{1+|r|} & \text { for } r \gg 1 \\ \frac{1}{\sqrt{1+|r|}} & \text { for } r \ll-1\end{cases}
$$


and also

$$
\left\|\frac{1}{z-k_{0}}\left(h^{2} W(x)+h^{2} A(x)^{2}\right)\right\|_{\infty} \leq C h^{2} \times\left\{\begin{array}{cl}
1 & \text { for } r \gg 1 \\
\frac{1}{1+|r|} & \text { for } r \ll-1 .
\end{array}\right.
$$

As in the proof of Lemma 8 , we can use (7.24) to bound $\left\|(z-k)^{-1}\right\|_{\infty}$. Moreover, it is not difficult to see that

$$
\left\|(z-k)^{-1}\right\|_{p} \leq C h^{-d / p} \times\left\{\begin{array}{cl}
r^{(d-2) /(2 p)} & \text { for } r \gg 1 \\
|r|^{-1+d /(2 p)} & \text { for } r \ll-1
\end{array}\right.
$$

for $d / 2<p \leq \infty$, generalizing (7.24). This follows from noting that $k$ can be replaced by $k_{0}$ for a bound, since their spectrum agrees up to $o(1)$. With $k_{0}$ in place of $k$, the result follows from evaluating the corresponding integral. In case $k$ is replaced by $-\bar{k}$, a similar bound holds, with the estimates for $r \gg 1$ and $r \ll-1$ interchanged.

For $d=3$, we hence end up with a function that decays like $|r|^{-10 / 3}$ for negative $r$ and $r^{-1}$ for positive $r$. Since we integrate against a function that decays exponentially for positive $r$ and increases linearly for negative $r$, the remaining contour integral is finite. We conclude that (7.32), multiplied by $f(\beta z)$ and integrated over $\Gamma$, is bounded by $C h^{1-d / 3}\|\Delta\|_{6}^{4}$. As in the proof of Lemma 8 , we can bound $\|\Delta\|_{6} \leq C\|\psi\|_{H^{1}(\mathcal{C})} h^{1-d / 6}\|t\|_{6}$. We have thus obtained a bound on the error made by replacing the first factor $(z-k)^{-1}$ in $I_{2}$ by $\left(z-k_{0}\right)^{-1}$.

In exactly the same way we proceed with the remaining factors $(z-k)^{-1}$ and $(z+\bar{k})^{-1}$ in $I_{2}$. The only difference is that $k$ might now be replaced by $k_{0}$ in the factors we have already treated, but this does not affect the bounds. Also the number of + and - terms is different, but we still get a decay of at least $|r|^{-7 / 3}$ for negative $r$, which is sufficient for the contour integral to converge.

The final result is that

$$
\begin{aligned}
& \left\|\int_{\Gamma} f(\beta z)\left[I_{2}-\frac{1}{z-k_{0}} \Delta \frac{1}{z+k_{0}} \Delta^{\dagger} \frac{1}{z-k_{0}} \Delta \frac{1}{z+k_{0}} \Delta^{\dagger} \frac{1}{z-k_{0}}\right] d z\right\|_{1} \\
& \leq O\left(h^{5-d}\right)\|\psi\|_{H^{1}(\mathcal{C})}^{4} .
\end{aligned}
$$

Proceeding as above, one shows that the remaining term on the left side of (7.36) is trace class. An explicit computation of the trace per unit volume yields the desired result.

Let us now look at the behavior of $F\left(p_{1}, p_{2}, p_{3}\right)$ for small arguments. We have

$$
[a, a, a,-a,-a]_{f}=\frac{1}{16 a} g_{1}(a)
$$

with $g_{1}$ defined in (2.10). With $a=\beta\left(q^{2}-\mu\right)$ we thus have

$$
F(0,0,0)=\frac{\beta^{3}}{16} \int_{\mathbb{R}^{d}} t(q)^{4} \frac{g_{1}\left(\beta\left(q^{2}-\mu\right)\right)}{q^{2}-\mu} \frac{d q}{(2 \pi)^{d}} .
$$

Lemma 10. For some constant $C$ depending on the $L^{6}{ }_{-n o r m}$ oft and its derivatives up to order four,

$$
\left|F\left(p_{1}, p_{2}, p_{3}\right)-F(0,0,0)\right| \leq C\left(p_{1}^{2}+p_{2}^{2}+p_{3}^{2}\right) .
$$


Proof. We will first show that $F$ is bounded. Using Hölder's inequality, we can bound

$$
\left|F\left(p_{1}, p_{2}, p_{3}\right)\right| \leq \beta^{4} \prod_{i=1}^{4} \frac{S_{i}+S_{i+1}}{2}
$$

where $S_{5}:=S_{1}$ and

$$
S_{i}^{4}=\int_{\mathbb{R}^{d}}\left|t\left(q+\sum_{j=1}^{i-1} p_{i}\right)\right|^{4}\left|\left[a_{3}, a_{3}, a_{1},-a_{2},-a_{0}\right]_{f}\right| \frac{d q}{(2 \pi)^{d}}
$$

for $1 \leq i \leq 4$. The last factor in the integrand is bounded by a constant times $\left(1+\beta\left(q+\sum_{j=1}^{i-1} p_{i}\right)^{2}\right)^{-1}$, for any $1 \leq i \leq 4$, as the bound (7.5) from Lemma 6 shows. A further application of Hölder's inequality thus shows that $S_{i}$ is bounded by $\|t\|_{6}$.

It is thus sufficient to consider the case $\left|p_{i}\right| \leq C$ for all $i \in\{1,2,3\}$. We write

$$
t(q+p)=t(q)+p \cdot \nabla t(q)+\int_{0}^{1}(p \cdot \nabla)^{2} t(q+s p)(1-s) d s
$$

and also, using partial fractions (or, equivalently, (7.2)),

$$
\begin{aligned}
& {\left[\beta\left((q+p)^{2}-\mu\right), a, b, c, d\right]_{f}} \\
& =\left[\beta\left(q^{2}-\mu\right), a, b, c, d\right]_{f}+\beta\left(2 p \cdot q+p^{2}\right)\left[\beta\left((q+p)^{2}-\mu\right), \beta\left(q^{2}-\mu\right), a, b, c, d\right]_{f} .
\end{aligned}
$$

We expand all these factors to second order in $p$ and, in particular, iterate (7.43) once more. Using the fact that the derivatives of $t$ are in $L^{6}$, as well as the decay bound (7.6) of Lemma 6, we can proceed as above, using Hölder's inequality, to arrive at the result. Note that all terms linear in $p$ integrate to zero since $t$ is reflection-symmetric.

We have

$$
\sum_{p_{1}, p_{2}, p_{3} \in(2 \pi \mathbb{Z})^{d}} \widehat{\psi}\left(p_{1}\right) \widehat{\psi}^{*}\left(p_{2}\right) \widehat{\psi}\left(p_{3}\right) \widehat{\psi}^{*}\left(-p_{1}-p_{2}-p_{3}\right)=\int_{\mathcal{C}}|\psi(x)|^{4} d x .
$$

With the aid of the Schwarz inequality for the $p_{1}$ sum and Sobolev's inequality, we can bound, similarly to (5.59)-(5.60),

$$
\sum_{p_{1}, p_{2}, p_{3} \in(2 \pi \mathbb{Z})^{d}} p_{1}^{2}\left|\widehat{\psi}^{*}\left(p_{1}\right) \widehat{\psi}^{*}\left(p_{2}\right) \widehat{\psi}\left(p_{3}\right) \widehat{\psi}\left(-p_{1}-p_{2}-p_{3}\right)\right| \leq C\|\psi\|_{H^{2}}\|\psi\|_{H^{1}}^{3} .
$$

Equal bounds hold with $p_{1}^{2}$ replaced by $p_{2}^{2}$ and $p_{3}^{2}$, respectively. Hence we arrive at the result

$$
\begin{aligned}
& \left.\left|\frac{1}{2 \pi i} \operatorname{Tr} \int_{\Gamma} f(\beta z) I_{2} d z-h^{4-d} F(0,0,0) \int_{\mathcal{C}}\right| \psi(x)\right|^{4} d x \mid \\
& \leq C h^{5-d}\|\psi\|_{H^{1}(\mathcal{C})}^{4}+C h^{6-d}\|\psi\|_{H^{2}}\|\psi\|_{H^{1}}^{3},
\end{aligned}
$$

with $F(0,0,0)$ given in (7.38). The term involving $F(0,0,0)$ gives rise to the last term in (2.14).

We are left with examining the contribution of $I_{1}$ to the integral in (7.11).

Lemma 11. The operator $\int_{\Gamma} f(\beta z) I_{1} d z$ is locally trace class. 
The proof of Lemma 11 is somewhat technical and will be given in Appendix B

Using the resolvent identity (7.29) we can write $I_{1}=I_{1}^{a}+I_{1}^{b}+I_{1}^{c}+I_{1}^{d}$, where

$$
I_{1}^{a}=\frac{1}{z-k_{0}} \Delta \frac{1}{z+k_{0}} \Delta^{\dagger} \frac{1}{z-k_{0}}
$$

and

$$
\begin{aligned}
I_{1}^{b}= & \frac{1}{z-k_{0}}\left(k-k_{0}\right) \frac{1}{z-k_{0}} \Delta \frac{1}{z+k_{0}} \Delta^{\dagger} \frac{1}{z-k_{0}} \\
& +\frac{1}{z-k_{0}} \Delta \frac{1}{z+k_{0}}\left(k_{0}-\bar{k}\right) \frac{1}{z+k_{0}} \Delta^{\dagger} \frac{1}{z-k_{0}} \\
& +\frac{1}{z-k_{0}} \Delta \frac{1}{z+k_{0}} \Delta^{\dagger} \frac{1}{z-k_{0}}\left(k-k_{0}\right) \frac{1}{z-k_{0}} .
\end{aligned}
$$

The part $I_{1}^{c}$ consists of 6 terms involving two $\left(k_{0}-k\right)$ factors. Explicitly,

$$
\begin{aligned}
I_{1}^{c}= & \frac{1}{z-k_{0}}\left(k-k_{0}\right) \frac{1}{z-k_{0}}\left(k-k_{0}\right) \frac{1}{z-k_{0}} \Delta \frac{1}{z+k_{0}} \Delta^{\dagger} \frac{1}{z-k_{0}} \\
& +\frac{1}{z-k_{0}} \Delta \frac{1}{z+k_{0}}\left(k_{0}-\bar{k}\right) \frac{1}{z+k_{0}}\left(k_{0}-\bar{k}\right) \frac{1}{z+k_{0}} \Delta^{\dagger} \frac{1}{z-k_{0}} \\
& +\frac{1}{z-k_{0}} \Delta \frac{1}{z+k_{0}} \Delta^{\dagger} \frac{1}{z-k_{0}}\left(k-k_{0}\right) \frac{1}{z-k_{0}}\left(k-k_{0}\right) \frac{1}{z-k_{0}} \\
& +\frac{1}{z-k_{0}}\left(k-k_{0}\right) \frac{1}{z-k_{0}} \Delta \frac{1}{z+k_{0}}\left(k_{0}-\bar{k}\right) \frac{1}{z+k_{0}} \Delta^{\dagger} \frac{1}{z-k_{0}} \\
& +\frac{1}{z-k_{0}}\left(k-k_{0}\right) \frac{1}{z-k_{0}} \Delta \frac{1}{z+k_{0}} \Delta^{\dagger} \frac{1}{z-k_{0}}\left(k-k_{0}\right) \frac{1}{z-k_{0}} \\
& +\frac{1}{z-k_{0}} \Delta \frac{1}{z+k_{0}}\left(k_{0}-\bar{k}\right) \frac{1}{z+k_{0}} \Delta^{\dagger} \frac{1}{z-k_{0}}\left(k-k_{0}\right) \frac{1}{z-k_{0}} .
\end{aligned}
$$

Finally, $I_{1}^{d}$ contains all the rest.

A straightforward computation of the trace and the contour integral shows that

$$
\frac{1}{2 \pi i} \operatorname{Tr} \int_{\Gamma} f(\beta z) I_{1}^{a} d z=h^{2-d} \sum_{p \in(2 \pi \mathbb{Z})^{d}}|\widehat{\psi}(p)|^{2} G(h p),
$$

where

$$
G\left(p_{1}\right)=\beta^{2} \int_{\mathbb{R}^{d}} \frac{\left(t\left(q+p_{1}\right)+t(q)\right)^{2}}{4}\left[a_{1}, a_{1},-a_{0}\right]_{f} \frac{d q}{(2 \pi)^{d}} .
$$

Here, we use the notation introduced in Lemma 9. Explicitly, $G(p)$ equals

$$
-\frac{\beta}{4} \int_{\mathbb{R}^{d}} \frac{(t(q+p)+t(q))^{2}}{4} \frac{\tanh \left(\frac{1}{2} \beta\left((q+p)^{2}-\mu\right)\right)+\tanh \left(\frac{1}{2} \beta\left(q^{2}-\mu\right)\right)}{(q+p)^{2}+q^{2}-2 \mu} \frac{d q}{(2 \pi)^{d}} .
$$

Lemma 12. The function $G$ in (7.49) is bounded, twice differentiable at zero, and

$$
\left|G(p)-G(0)-\frac{1}{2}(p \cdot \nabla)^{2} G(0)\right| \leq C|p|^{4}
$$

for some constant $C$ depending only on $\int_{\mathbb{R}^{d}} t(q)^{2}\left(1+q^{2}\right)^{-1} d q$ and the $L^{6}$-norm of $t$ and its derivatives up to order four.

When inserted into (7.48), the term $C|p|^{4}$ thus yields an error of the order $h^{6-d}\|\psi\|_{H^{2}}^{2}$. 
Proof. It follows from (7.5) of Lemma 6 that

$$
\left|\left[a_{1}, a_{1},-a_{0}\right]_{f}\right| \leq \frac{C}{1+q^{2}+(p+q)^{2}} ;
$$

hence $G$ is bounded by $\int_{\mathbb{R}^{d}} t(q)^{2}\left(1+q^{2}\right)^{-1} d q$, which is finite by our assumption (2.4) on $t$. To prove (7.51), it thus suffices to consider the case $|p| \leq C$.

We expand $t(q+p)$ up to fourth order in $p$. Similarly, we write $a_{1}=a_{0}+\beta\left(2 p_{1}\right.$. $\left.q+p_{1}^{2}\right)$ and expand the brackets, using the resolvent identity. The decay of the resulting expression for large $q$ is governed by (7.6). Using in addition that $t$ is reflection-symmetric and satisfies (2.3), the result follows in a straightforward way from Hölder's inequality. We omit the details.

Recall the definition of the $g_{i}$ in (2.9) -(2.11). We have

$$
G(0)=-\frac{\beta^{2}}{4} \int_{\mathbb{R}^{d}} t(q)^{2} g_{0}\left(\beta\left(q^{2}-\mu\right)\right) \frac{d q}{(2 \pi)^{d}},
$$

which gives rise to the expression in (2.13). Using integration by parts,

$$
\begin{aligned}
(p \cdot \nabla)^{2} G(0)= & -\frac{\beta^{2}}{8} \int_{\mathbb{R}^{d}} t(q)\left[(p \cdot \nabla)^{2} t\right](q) g_{0}\left(\beta\left(q^{2}-\mu\right)\right) \frac{d q}{(2 \pi)^{d}} \\
& +\frac{\beta^{4}}{4} \int_{\mathbb{R}^{d}}(p \cdot q)^{2} t(q)^{2} g_{2}\left(\beta\left(q^{2}-\mu\right)\right) \frac{d q}{(2 \pi)^{d}} \\
& +\frac{\beta^{3} p^{2}}{8} \int_{\mathbb{R}^{d}} t(q)^{2} g_{1}\left(\beta\left(q^{2}-\mu\right)\right) \frac{d q}{(2 \pi)^{d}} .
\end{aligned}
$$

We proceed with $I_{1}^{b}$ and insert (7.31). We treat successively the terms proportional to $W$, to $A^{2}$, and to $A$. After doing the contour integration and evaluating the trace per unit volume, the six terms proportional to $W$ are

$$
h^{4-d} \sum_{p_{1}, p_{2} \in(2 \pi \mathbb{Z})^{d}} \widehat{\psi}^{*}\left(p_{1}\right) \widehat{\psi}\left(p_{2}\right) \widehat{W}\left(-p_{1}-p_{2}\right) L\left(h p_{1}, h p_{2}\right),
$$

where

$$
L\left(p_{1}, p_{2}\right)=\beta^{3} \int_{\mathbb{R}^{d}} L\left(p_{1}, p_{2}, q\right) \frac{d q}{(2 \pi)^{d}}
$$

and

$$
\begin{aligned}
L\left(p_{1}, p_{2}, q\right)= & \frac{1}{4}\left(t(q)+t\left(q+p_{1}\right)\right)\left(t\left(q+p_{1}\right)+t\left(q+p_{1}+p_{2}\right)\right) \\
& \times\left(\left[a_{0}, a_{0}, a_{2},-a_{1}\right]_{f}+\left[a_{0}, a_{2}, a_{2},-a_{1}\right]_{f}+\left[a_{0}, a_{2},-a_{1},-a_{1}\right]_{f}\right) .
\end{aligned}
$$

Since

$$
[a, a, a,-a]_{f}=\frac{1}{8} g_{1}(a)
$$

we have

$$
L(0,0)=\frac{\beta^{3}}{4} \int_{\mathbb{R}^{d}} t(q)^{2} g_{1}\left(\beta\left(q^{2}-\mu\right)\right) \frac{d q}{(2 \pi)^{d}} .
$$


In the same way as in Lemma 12, one proves that

Lemma 13. The function $L$ is bounded, with

$$
\left|L\left(p_{1}, p_{2}\right)-L(0,0)\right| \leq C\left(p_{1}^{2}+p_{2}^{2}\right)
$$

for some constant $C>0$.

The contribution of this term is thus

$$
h^{4-d} L(0,0) \int_{\mathcal{C}} W(x)|\psi(x)|^{2} d x+O\left(h^{6-d}\right)\|\psi\|_{H^{2}}^{2},
$$

where we have used a simple Schwarz inequality to bound the error term, as well as the fact that $W \in L^{2}(\mathcal{C})$ and that $|\widehat{\psi}(p)| \leq C\|\psi\|_{H^{2}}$ for all $p \in(2 \pi \mathbb{Z})^{d}$.

We obtain the same contribution with $A(x)^{2}$ in place of $W(x)$. The terms linear in $A$ are given by

$h^{3-d} \sum_{p_{1}, p_{2} \in(2 \pi \mathbb{Z})^{d}} \widehat{\psi}^{*}\left(p_{1}\right) \widehat{\psi}\left(p_{2}\right) \widehat{A}\left(-p_{1}-p_{2}\right) \cdot \int_{\mathbb{R}^{d}} L\left(h p_{1}, h p_{2}, q\right)\left(2 q+h p_{1}+h p_{2}\right) \frac{d q}{(2 \pi)^{d}}$.

For small $h$, we have

$$
\int_{\mathbb{R}^{d}} L\left(h p_{1}, h p_{2}, q\right)\left(2 q+h p_{1}+h p_{2}\right) \frac{d q}{(2 \pi)^{d}}=h \mathbb{K}\left(p_{2}-p_{1}\right)+O\left(h^{2}\right),
$$

where $\mathbb{K}$ is a symmetric $d \times d$ matrix with entries

$$
\mathbb{K}_{i j}=\frac{1}{2} L(0,0) \delta_{i j}+\frac{\beta^{4}}{4} \int_{\mathbb{R}^{d}} t(q)^{2} q_{i} q_{j} g_{2}\left(\beta\left(q^{2}-\mu\right)\right) \frac{d q}{(2 \pi)^{d}} .
$$

Hence the leading term is

$$
h^{4-d} 2 \operatorname{Re} \int_{\mathcal{C}} \psi(x)^{*} A(x) \cdot \mathbb{K}(-i \nabla \psi(x)) d x .
$$

The remainder can be bounded by

$$
C h^{5-d} \sum_{p_{1}, p_{2} \in(2 \pi \mathbb{Z})^{d}}\left|\widehat{\psi}^{*}\left(p_{1}\right) \widehat{\psi}\left(p_{2}\right) \widehat{A}\left(-p_{1}-p_{2}\right)\right|\left(p_{1}^{2}+p_{2}^{2}\right) \leq C h^{5-d}\|\psi\|_{H^{1}(\mathcal{C})}^{2}
$$

using that $|\widehat{A}(p)||p|$ is summable by assumption.

We proceed with $I_{1}^{c}$. First, there is the contribution of the terms linear in $A$ in both factors $k-k_{0}$. These we have to calculate explicitly to leading order in $h$, and then bound the remainder. They contribute

$$
h^{4-d} \frac{\beta^{4}}{2} \sum_{i, j=1}^{d} \int_{\mathcal{C}}|\psi(x)|^{2} A_{i}(x) A_{j}(x) d x \int_{\mathbb{R}^{d}} t(q)^{2} q_{i} q_{j} g_{2}\left(\beta\left(q^{2}-\mu\right)\right) \frac{d q}{(2 \pi)^{d}}
$$

plus terms of order $O\left(h^{5-d}\right)\|\psi\|_{H^{1}}^{2}$ and higher. In combination, (7.61) for $W$ and $A^{2},(7.67)$ and (7.54) give all the terms in (2.14) except for the last, which came from $F(0,0,0)$ in (7.38).

For the other terms, we use (7.33) and (7.34), and bound all expressions involving $z$ with $\infty$-norms. We always get enough decay in either the positive or the negative $z$-direction. Only decay in one direction is needed, as $z$ can be replaced by $-z$, which follows from the fact that the resulting expressions are zero if $f(\beta z)$ is replaced by $f(\beta z)-f(-\beta z)=\beta z$. (Compare with the discussion at the end of the proof of 
Lemma 11, in Appendix B] The same is true for $I_{1}^{d}$. We omit the details. The final result is then that

$$
\frac{1}{2 \pi i} \operatorname{Tr} \int_{\Gamma} f(\beta z)\left(I_{1}^{c}+I_{1}^{d}\right) d z=\left(\mathbf{7 . 6 7 )}+O\left(h^{5-d}\right)\|\psi\|_{H^{1}}^{2} .\right.
$$

This completes the proof of Theorem 2

\section{Proof of Theorem 3}

As in the proof of Theorem 2, we restrict our attention to the case $d=3$ and leave the cases $d=1$ and $d=2$ to the reader.

Since the function

$$
\rho(z)=\frac{1}{1+e^{z}}
$$

is analytic in the strip $|\operatorname{Im} z|<\pi$, we can write $\alpha_{\Delta}$ with the aid of a contour integral representation as

$$
\alpha_{\Delta}=\frac{1}{2 \pi i} \int_{\Gamma} \rho(\beta z)\left[\frac{1}{z-H_{\Delta}}\right]_{12} d z
$$

where $\Gamma$ is the contour $\operatorname{Im} z= \pm \pi /(2 \beta)$, and $[\cdot]_{12}$ denotes the upper off-diagonal entry of an operator-valued $2 \times 2$ matrix. The integral in (8.2) has to be suitably understood as a weak limit, similarly to (7.10).

Using the resolvent identity and the definitions of $\Delta$ and $\varphi$ in (2.1) and (2.17), respectively, we find that

$$
\alpha_{\Delta}=\frac{h}{2}(\psi(x) \varphi(-i h \nabla)+\varphi(-i h \nabla) \psi(x))+\sum_{j=1}^{3} \eta_{j},
$$

where

$$
\begin{aligned}
& \eta_{1}=\frac{h}{4 \pi i} \int_{\Gamma} \rho(\beta z)\left(\frac{1}{z-k_{0}}\left[\psi, k_{0}\right] \frac{t}{z^{2}-k_{0}^{2}}+\frac{t}{z^{2}-k_{0}^{2}}\left[\psi, k_{0}\right] \frac{1}{z+k_{0}}\right) d z \\
& \eta_{2}=\frac{1}{2 \pi i} \int_{\Gamma} \rho(\beta z) \frac{1}{z-k_{0}}\left(\left(k-k_{0}\right) \frac{1}{z-k} \Delta+\Delta \frac{1}{z+k_{0}}\left(k_{0}-\bar{k}\right)\right) \frac{1}{z+\bar{k}} d z
\end{aligned}
$$

and

$$
\eta_{3}=\frac{1}{2 \pi i} \int_{\Gamma} \rho(\beta z) \frac{1}{z-k} \Delta \frac{1}{z+\bar{k}} \Delta^{\dagger} \frac{1}{z-k} \Delta\left[\frac{1}{z-H_{\Delta}}\right]_{22} d z
$$

with $t$ being short for the operator $t(-i h \nabla)$. In the following three lemmas we give bounds on these three operators.

\section{Lemma 14.}

$$
\left\|\eta_{1}\right\|_{H^{1}} \leq C h^{3-d / 2}\|\psi\|_{H^{2}(\mathcal{C})}
$$

Proof. The $H^{1}$-norm of $\eta_{1}$ is given by

$$
\left\|\eta_{1}\right\|_{H^{1}}^{2}=h^{2-d} \sum_{p \in(2 \pi \mathbb{Z})^{d}}|\widehat{\psi}(p)|^{2} J(h p),
$$


where

$$
\begin{aligned}
J(p)=\frac{\beta^{4}}{4} \int_{\mathbb{R}^{d}} & \left((q+p)^{2}-q^{2}\right)^{2}\left(1+(q+p)^{2}\right) \\
& \times\left|t(q)\left[a_{0},-a_{0}, a_{1}\right]_{\rho}+t(p+q)\left[-a_{0}, a_{1},-a_{1}\right]_{\rho}\right|^{2} \frac{d q}{(2 \pi)^{d}} .
\end{aligned}
$$

Here, we use again the notation $[\cdot]_{\rho}$ for the divided differences (7.1)-(7.2), and $a_{0}=\beta\left(q^{2}-\mu\right)$ and $a_{1}=\beta\left((q+p)^{2}-\mu\right)$, as in the proof of Theorem 2. Since

$$
[a, a,-a]_{\rho}=-[a,-a,-a]_{\rho}=\frac{\rho(-a)-\rho(a)}{4 a^{2}}+\frac{\rho^{\prime}(a)}{2 a}=\frac{1+2 a e^{a}-e^{2 a}}{4 a^{2}\left(1+e^{a}\right)^{2}},
$$

the integrand in (8.9) vanishes like the fourth power of $p$ for small $p$. In fact, using Lemma 6 and our assumptions on the regularity of $t$ it is easy to see that $J(p) \leq C|p|^{4}$, which yields the desired bound.

We proceed with a bound on the $H^{1}$-norm of $\eta_{2}$.

\section{Lemma 15.}

$$
\left\|\eta_{2}\right\|_{H^{1}} \leq C h^{3-d / 2}\|\psi\|_{H^{1}(\mathcal{C})} .
$$

Proof. We split $\eta_{2}$ into three parts, $\eta_{2}^{a}, \eta_{2}^{b}$ and $\eta_{2}^{c}$, where

$$
\begin{aligned}
\eta_{2}^{a}=-\frac{h^{2}}{2 \pi} \int_{\Gamma} \rho(\beta z) \frac{1}{z-k_{0}}( & (\nabla \cdot A+A \cdot \nabla) \frac{1}{z-k_{0}} \Delta \\
& \left.+\Delta \frac{1}{z+k_{0}}(\nabla \cdot A+A \cdot \nabla)\right) \frac{1}{z+k_{0}} d z
\end{aligned}
$$

$$
\eta_{2}^{b}=\frac{h^{2}}{2 \pi i} \int_{\Gamma} \rho(\beta z) \frac{1}{z-k_{0}}\left((-i \nabla \cdot A-i h A \cdot \nabla) \frac{1}{z-k_{0}}\left(k-k_{0}\right) \frac{1}{z-k} \Delta\right.
$$

$$
\begin{aligned}
& +(-i \nabla \cdot A-i A \cdot \nabla) \frac{1}{z-k_{0}} \Delta \frac{1}{z+k_{0}}\left(k_{0}-\bar{k}\right) \\
& \left.+\Delta \frac{1}{z+k_{0}}(-i \nabla \cdot A-i A \cdot \nabla) \frac{1}{z+k_{0}}\left(k_{0}-\bar{k}\right)\right) \frac{1}{z+\bar{k}} d z
\end{aligned}
$$

and

$$
\eta_{2}^{c}=\frac{h^{2}}{2 \pi i} \int_{\Gamma} \rho(\beta z) \frac{1}{z-k_{0}}\left(\left(W+|A|^{2}\right) \frac{1}{z-k} \Delta-\Delta \frac{1}{z+k_{0}}\left(W+|A|^{2}\right)\right) \frac{1}{z+\bar{k}} .
$$

We start by considering the first term in $\eta_{2}^{c}$. The square of the $H^{1}$-norm of the integrand can be bounded by

$$
\begin{gathered}
\operatorname{Tr}\left[\frac{1-h^{2} \nabla^{2}}{\left|z-k_{0}\right|^{2}}\left(W+|A|^{2}\right) \frac{1}{z-k} \Delta \frac{1}{|z+\bar{k}|^{2}} \Delta^{\dagger} \frac{1}{\bar{z}-k}\left(W+|A|^{2}\right)\right] \\
\leq\|\Delta\|_{6}^{2}\left\|W+|A|^{2}\right\|_{\infty}^{2}\left\|\frac{1-h^{2} \nabla^{2}}{\left|z-k_{0}\right|^{2}}\right\|_{\infty}\left\|(z-k)^{-1}\right\|_{\infty}^{2}\left\|(z+\bar{k})^{-1}\right\|_{3}^{2}
\end{gathered}
$$

The latter expression decays like $|z|^{-3+(d-2) / 3}$ for negative Re $z$. Hence the resulting contour integral is finite, and we arrive at the bound $C h^{2-d / 3}\|\Delta\|_{6} \leq$ $C h^{3-d / 2}\|\psi\|_{H^{1}(\mathcal{C})}$ for the $H^{1}$-norm. 
For the second term in $\eta_{2}^{c}$ we proceed similarly. It is important to first notice that

$$
\frac{1}{1+e^{\beta z}}=1-\frac{1}{1+e^{-\beta z}},
$$

however, and that the 1 does not contribute anything but integrates to zero. Proceeding as above, we conclude that

$$
\left\|\eta_{2}^{c}\right\|_{H^{1}}^{2} \leq C h^{6-d}\|\psi\|_{H^{1}(\mathcal{C})}^{2} .
$$

The $H^{1}$-norm of $\eta_{2}^{b}$ can be bounded in essentially the same way, and we omit the details. The result is that also

$$
\left\|\eta_{2}^{b}\right\|_{H^{1}}^{2} \leq C h^{6-d}\|\psi\|_{H^{1}(\mathcal{C})}^{2}
$$

holds.

At first sight, the $H^{1}$-norm of $\eta_{2}^{a}$ appears to be of order $h^{2-d / 2}$ instead of $h^{3-d / 2}$. Note, however, that if we commute $A(x)$ and $\psi(x)$ to the left in both terms, the resulting integral is zero due to the antisymmetry (8.10). Hence $\eta_{2}^{a}$ can be written as an integral over sums of various terms involving commutators of $A(x)$ and $\psi(x)$ with functions of $-i \nabla$, and hence an additional factor of $h$ is gained. In fact, the $H^{1}$-norm of $\eta_{2}^{a}$ can be written as

$$
\left\|\eta_{2}^{a}\right\|_{H^{1}}^{2}=h^{4-d} \sum_{p_{1}, p_{2}, p_{3} \in(2 \pi \mathbb{Z})^{d}} \widehat{\psi}\left(p_{1}\right) \widehat{\psi}^{*}\left(p_{2}\right) \widehat{A}\left(p_{3}\right) \cdot \mathbb{M}\left(h p_{1}, h p_{2}, h p_{3}\right) \widehat{A}\left(-p_{1}-p_{2}-p_{3}\right),
$$

where $\mathbb{M}$ is a $d \times d$ matrix-valued function which can easily be calculated explicitly. An analysis as in the proof of Lemma 14, using the antisymmetry (8.10), shows that

$$
\left\|\mathbb{M}\left(p_{1}, p_{2}, p_{3}\right)\right\| \leq C\left(p_{1}^{2}+p_{2}^{2}+p_{3}^{2}\right) .
$$

Under our assumption that $|\widehat{A}(p)|(1+|p|)$ is summable, this implies the result

$$
\left\|\eta_{2}^{a}\right\|_{H^{1}} \leq C h^{3-d / 2}\|\psi\|_{H^{1}(\mathcal{C})} .
$$

Finally we bound the $H^{1}$-norm of $\eta_{3}$.

\section{Lemma 16.}

$$
\left\|\eta_{3}\right\|_{H^{1}} \leq C h^{3-d / 2}\|\psi\|_{H^{1}(\mathcal{C})}^{3} .
$$

Proof. Since $H_{\Delta}$ is selfadjoint, $\left[\left(z-H_{\Delta}\right)^{-1}\right]_{22}$ is bounded by $2 \beta / \pi$ for $z \in \Gamma$. Using Hölder's inequality, we can bound the square of the $H^{1}$-norm of the integrand by

$$
\frac{4 \beta^{2}}{\pi^{2}}\|\Delta\|_{6}^{6}\left\|(z-k)^{-1}\right\|_{\infty}^{2}\left\|(z+k)^{-1}\right\|_{\infty}^{2}\left\|\sqrt{1-h^{2} \nabla^{2}}(z-k)^{-1}\right\|_{\infty}^{2} .
$$

This expression decays like $|z|^{-3}$ for $\operatorname{Re} z<0$, and like $|z|^{-1}$ for $\operatorname{Re} z>0$. Hence

$$
\left\|\eta_{3}\right\|_{H^{1}}^{2} \leq C\|\Delta\|_{6}^{6} \leq C h^{6-d}\|\psi\|_{H^{1}(\mathcal{C})}^{6} .
$$

This completes the proof of Theorem 3 . 


\section{Appendix A. Properties of $\alpha_{0}$}

Recall the definition of $K_{T}$ in (1.6) and recall that $\alpha_{0}$ denotes the eigenfunction of $K_{T_{c}}+V$ corresponding to the eigenvalue zero, which we assume to be simple. We assume that $T_{c}>0$, and that $V \in L^{p}\left(\mathbb{R}^{d}\right)$, with $p=1$ for $d=1, p>1$ for $d=2$ and $p=3 / 2$ for $d=3$. There is no parameter $h$ in the definition of $K_{T}$; in other words, $h=1$ in this section.

\section{Proposition 1.}

$$
e^{\kappa|x|} \sqrt{|V|} \alpha_{0} \in L^{2}\left(\mathbb{R}^{d}\right)
$$

for any $\kappa<\kappa_{c}:=\operatorname{Im} \sqrt{\mu+i \pi T_{c}}$.

In three dimensions, this proposition also holds for $\kappa=\kappa_{c}$, in fact.

The key to prove Proposition 1 is to estimate the integral kernel of $K_{T}^{-1}$. It turns out to have the same behavior as the one for $\left(-\nabla^{2}+\kappa_{c}^{2}\right)^{-1}$, both at the origin and at infinity. Hence we find it convenient to state our bound in terms of $G(x-y ; \lambda)$, the Green's function of $-\nabla^{2}-\lambda$ for $\lambda \in \mathbb{C} \backslash \mathbb{R}_{+}$. Note that $G(x, \lambda)>0$ for $\lambda<0$.

Lemma 17. For some constant $C_{\mu / T}$ depending on $\mu / T$, the integral kernel of $K_{T}^{-1}$ satisfies

$$
\left|K_{T}^{-1}(x-y)\right| \leq C_{\mu / T} G\left(x-y ;-(\operatorname{Im} \sqrt{\mu+i \pi T})^{2}\right) .
$$

Proof. We start with the series representation

$$
\frac{\tanh z}{z}=\sum_{n=1}^{\infty} \frac{2}{\left(n-\frac{1}{2}\right)^{2} \pi^{2}+z^{2}}
$$

which implies that

$$
K_{T}^{-1}=\sum_{n=1}^{\infty} \frac{4 T}{\left(n-\frac{1}{2}\right)^{2}(2 \pi T)^{2}+\left(-\nabla^{2}-\mu\right)^{2}} .
$$

We can rewrite this as

$$
K_{T}^{-1}=\frac{2}{\pi} \sum_{n=1}^{\infty} \frac{1}{n-\frac{1}{2}} \operatorname{Im} \frac{1}{-\nabla^{2}-\mu-i\left(n-\frac{1}{2}\right) 2 \pi T} .
$$

Hence

$$
K_{T}^{-1}(x-y)=\frac{2}{\pi} \sum_{n=1}^{\infty} \frac{1}{n-\frac{1}{2}} \operatorname{Im} G\left(x-y ; \mu+i\left(n-\frac{1}{2}\right) 2 \pi T\right) .
$$

The result now follows easily using the explicit behavior of the Green's function $G$ at 0 and at infinity.

Lemma 18. Let $L$ be an integral operator with integral kernel bounded as

$$
|L(x, y)| \leq A e^{-\kappa|x-y|} G(x-y ;-e)
$$

for $\kappa>0$ and $e>0$ (or $e \geq 0$ in $d=3$ ). Then there is a finite constant $C_{p, d}$ such that for any $U_{1}, U_{2} \in L^{2 p}\left(\mathbb{R}^{d}\right)$,

$$
\left\|e^{\kappa|x|} U_{1} L U_{2} e^{-\kappa|x|}\right\| \leq A C_{p, d} e^{d /(2 p)-1}\left\|U_{1}\right\|_{2 p}\left\|U_{2}\right\|_{2 p},
$$

where $p \geq 1$ in $d=1, p>1$ in $d=2$ and $p \geq 3 / 2$ in $d=3$. 
Proof. For $\psi \in L^{2}\left(\mathbb{R}^{d}\right)$,

$$
\begin{aligned}
& \left\|e^{\kappa|x|} U_{1} L U_{2} e^{-\kappa|x|} \psi\right\|_{2}^{2} \\
& \leq \int_{\mathbb{R}^{3 d}}|\psi(y)| e^{-\kappa|y|}\left|U_{2}(y)\right||L(x, y)|\left|U_{1}(x)\right|^{2} e^{2 \kappa|x|} \\
& \times\left|L\left(x, y^{\prime}\right)\right|\left|U_{2}\left(y^{\prime}\right)\right| e^{-\kappa\left|y^{\prime}\right|}\left|\psi\left(y^{\prime}\right)\right| d y d y^{\prime} d x \\
& \leq A^{2} \int_{\mathbb{R}^{3 d}}|\psi(y)|\left|U_{2}(y)\right| G(x-y ;-e)\left|U_{1}(x)\right|^{2} G\left(x-y^{\prime} ;-e\right)\left|U_{2}\left(y^{\prime}\right)\right|\left|\psi\left(y^{\prime}\right)\right| d y d y^{\prime} d x \\
& =A^{2}\left\|| U _ { 1 } | ( - \nabla ^ { 2 } + e ) ^ { - 1 } \left|U_{2}\|\psi \mid\|_{2}^{2},\right.\right.
\end{aligned}
$$

where we used the triangle inequality $2|x|-|y|-\left|y^{\prime}\right| \leq|x-y|+\left|x-y^{\prime}\right|$. The result now follows from the fact that the operator norm of $\left|U_{1}\right|\left(-\nabla^{2}+e\right)^{-1}\left|U_{2}\right|$ is bounded by a constant times $e^{d /(2 p)-1}\left\|U_{1}\right\|_{2 p}\left\|U_{2}\right\|_{2 p}$, as can be seen by an application of the Hausdorff-Young inequality (for $e>0$ ) or the Hardy-Littlewood-Sobolev inequality (for $e=0$ and $d=3$ ) and Hölder's inequality.

Proof of Proposition 1, The function $\phi=\sqrt{|V|} \alpha_{0}$ is in $L^{2}\left(\mathbb{R}^{d}\right)$ (because of the relative form-boundedness of $V$ ) and satisfies

$$
\phi=-\sqrt{|V|} \frac{1}{K_{T_{c}}} \sqrt{V} \phi
$$

where $\sqrt{V}:=V / \sqrt{|V|}$. For given $R>0$, we decompose $\phi=\phi_{1}+\phi_{2}$, where $\phi_{2}=\phi \chi_{\{|x|>R\}}$. Introducing $U_{1}=-\chi_{\{|x|>R\}} \sqrt{|V|}$ and $U_{2}=\chi_{\{|x|>R\}} \sqrt{V}$, we have

$$
\phi_{2}=U_{1} \frac{1}{K_{T_{c}}} U_{2} \phi_{2}+f
$$

where

$$
f=U_{1} \frac{1}{K_{T_{c}}} \sqrt{V} \phi_{1} .
$$

We shall now use that the Green's function of the Laplacian has the property

$$
G\left(x-y ;-\kappa^{2}\right) \leq C_{\epsilon / \kappa} e^{-(\kappa-e)|x-y|} G\left(x-y ;-e^{2}\right)
$$

for $0<e \leq \kappa$. In combination with Lemma 17 and Lemma 18, this implies that for $\kappa<\kappa_{c}$, the operator

$$
e^{\kappa|x|} U_{1} \frac{1}{K_{T_{c}}} \sqrt{V} e^{-\kappa|x|}
$$

is bounded. Since $e^{\kappa|x|}\left|\phi_{1}\right| \leq e^{\kappa R}\left|\phi_{1}\right|$, we conclude that $e^{\kappa|x|} f \in L^{2}\left(\mathbb{R}^{d}\right)$. Similarly, we observe that

$$
\left\|e^{\kappa|x|} U_{1} \frac{1}{K_{T_{c}}} U_{2} e^{-\kappa|x|}\right\| \leq C\left\|V \chi_{\{|x|>R\}}\right\|_{p},
$$

with $p$ as in Assumption 1, The latter expression is less than one for $R$ large enough. Hence

$$
e^{\kappa|x|} \phi_{2}=\left(1-e^{\kappa|x|} U_{1} \frac{1}{K_{T_{c}}} U_{2} e^{-\kappa|x|}\right)^{-1} e^{\kappa|x|} f
$$


is an element of $L^{2}\left(\mathbb{R}^{d}\right)$. Since obviously also $e^{\kappa|x|} \phi_{1} \in L^{2}\left(\mathbb{R}^{d}\right)$, this concludes the proof.

We shall use the result of Proposition 1 in the following way. Recall that $t$ was defined in (1.17) as twice the Fourier transform of $K_{T_{c}} \alpha_{0}=-V \alpha_{0}$. The following proposition collects all the regularity properties of $t$ and $\alpha_{0}$ that we need in the proof of Theorem 1 .

Proposition 2. The function $t$ in (1.17), together with all its derivatives, is a function in $L^{q}\left(\mathbb{R}^{d}\right)$, with $q=2 p /(p-1)$, i.e., $q=\infty$ for $d=1,2<q<\infty$ for $d=2$ and $q=6$ for $d=3$. We also have that

$$
\int_{\mathbb{R}^{d}} \frac{\left|\partial^{\gamma} t(p)\right|^{2}}{1+p^{2}} d p=4\left\langle x^{\gamma} \sqrt{|V|} \alpha_{0}\left|\sqrt{V} \frac{1}{1-\nabla^{2}} \sqrt{V}\right| x^{\gamma} \sqrt{|V|} \alpha_{0}\right\rangle<\infty
$$

and that $\int_{\mathbb{R}^{d}}\left(\left|x^{\gamma} \nabla \alpha_{0}(x)\right|^{2}+\left|x^{\gamma} \alpha_{0}(x)\right|^{2}\right) d x<\infty$ for all $\gamma \in \mathbb{N}_{0}^{d}$.

Proof. The $L^{q}$ property follows easily from the Hausdorff-Young inequality 20, Thm. 5.7] and the Hölder inequality,

$$
\left\|\partial^{\gamma} t\right\|_{2 p /(p-1)} \leq 2\left\|x^{\gamma} V \alpha_{0}\right\|_{2 p /(p+1)} \leq 2\|V\|_{p}^{1 / 2}\left\|x^{\gamma} \sqrt{|V|} \alpha_{0}\right\|_{2}
$$

using Proposition 1 and our assumption that $V \in L^{p}$.

The boundedness of (A.17) follows again from Proposition 1 and the fact that the operator on the right side is bounded by our assumption on $V$. Finally, the last statement follows easily from (A.17) writing the integral in Fourier space and using that $\widehat{\alpha}_{0}(p)=t(p) /\left(2 K_{T_{c}}(p)\right)$, where $K_{T_{c}}(p)$ denotes the function obtained by replacing $-\nabla^{2}$ by $p^{2}$ in (1.6).

For $d=3$, one could even drop the 1 in $1-\nabla^{2}$ and $1+p^{2}$ in (A.17); boundedness of the operator follows from the Hardy-Littlewood-Sobolev inequality.

\section{Appendix B. Proof of Lemma 11}

In this appendix, we shall give the proof that the operator

$$
B=\int_{\Gamma} f(\beta z) \frac{1}{z-k} \Delta \frac{1}{z+\bar{k}} \Delta^{\dagger} \frac{1}{z-k} d z
$$

is locally trace class. As in the proof of Theorem 2, we will focus our attention on the case $d=3$. Using the resolvent identity, we split $B$ into three parts, $B=\sum_{i=1}^{3} B_{i}$, with

$$
\begin{gathered}
B_{1}=\int_{\Gamma} f(\beta z) \frac{1}{z-k_{0}} \Delta \frac{1}{z+\bar{k}} \Delta^{\dagger} \frac{1}{z-k_{0}} d z \\
B_{2}=\int_{\Gamma} f(\beta z) \frac{1}{z-k_{0}}\left(k-k_{0}\right) \frac{1}{z-k} \Delta \frac{1}{z+\bar{k}} \Delta^{\dagger} \frac{1}{z-k} d z,
\end{gathered}
$$

and

$$
B_{3}=\int_{\Gamma} f(\beta z) \frac{1}{z-k_{0}} \Delta \frac{1}{z+\bar{k}} \Delta^{\dagger} \frac{1}{z-k}\left(k-k_{0}\right) \frac{1}{z-k_{0}} d z .
$$

We shall show that each individual piece is locally trace class.

We shall start with $B_{2}$. Using Hölder's inequality, the trace norm of the integrand is bounded by $|f(\beta z)|$ times

$$
\left\|\frac{1}{z-k_{0}}\left(k-k_{0}\right)\right\|_{\infty}\|\Delta\|_{6}^{2}\left\|(z-k)^{-1}\right\|_{p}\left\|\left(z-k_{0}\right)^{-1}\right\|_{p}\left\|(z+\bar{k})^{-1}\right\|_{q}
$$


with $2 / p+1 / q=2 / 3$. We shall choose $q^{-1}=2 / 3-\epsilon$ for some $0<\epsilon<1 / 6$. The bounds (7.33) - (7.35) then imply that (B.5) increases as $|z|^{1 / 2-\epsilon}$ for $\operatorname{Re} z>0$, and decreases like $|z|^{-13 / 6+\epsilon}$ for $\operatorname{Re} z<0$. Since $|f(\beta z)|$ decays exponentially for $\operatorname{Re} z>0$ and increases linearly for $\operatorname{Re} z<0$, the contour integral is absolutely convergent. This shows that $B_{2}$ is locally trace class. In exactly the same way, one shows that also $B_{3}$ is locally trace class.

For the remaining term $B_{1}$ it is not possible to take the trace norm of the integrand and obtain a convergent integral. Hence we have to argue differently. We again use the resolvent identity to write

$$
\begin{aligned}
\frac{1}{z+\bar{k}}= & \frac{1}{z+k_{0}}+\frac{1}{z+k_{0}}\left(k_{0}-\bar{k}\right) \frac{1}{z+k_{0}}+\frac{1}{z+k_{0}}\left(k_{0}-\bar{k}\right) \frac{1}{z+k_{0}}\left(k_{0}-\bar{k}\right) \frac{1}{z+k_{0}} \\
& +\frac{1}{z+k_{0}}\left(k_{0}-\bar{k}\right) \frac{1}{z+k_{0}}\left(k_{0}-\bar{k}\right) \frac{1}{z+k_{0}}\left(k_{0}-\bar{k}\right) \frac{1}{z+\bar{k}}
\end{aligned}
$$

and, correspondingly, write $B_{1}=B_{1}^{a}+B_{1}^{b}+B_{1}^{c}+B_{1}^{d}$.

We start with $B_{1}^{a}$. Recall that $\Delta$ is the sum of two terms,

$$
\Delta=-\frac{h}{2}(\psi(x) t(-i h \nabla)+t(-i h \nabla) \psi(x))
$$

By expanding $\psi$ in a Fourier series, we can use the triangle inequality to bound

$$
\left\|B_{1}^{a}\right\|_{1} \leq \frac{h^{2}}{4} \sum_{p_{1}, p_{2} \in(2 \pi \mathbb{Z})^{d}}\left|\widehat{\psi}\left(p_{1}\right)\left\|\widehat{\psi}\left(p_{2}\right) \mid\right\| D_{p_{1}, p_{2}} \|_{1},\right.
$$

where

$$
\begin{aligned}
D_{p_{1}, p_{2}}=\int_{\Gamma} & f(\beta z) e^{-i p_{1} \cdot x} \frac{1}{z-k_{0}}\left(e^{i p_{1} \cdot x} t(-i h \nabla)+t(-i h \nabla) e^{i p_{1} \cdot x}\right) \frac{1}{z+k_{0}} \\
& \times\left(t(-i h \nabla) e^{-i p_{2} \cdot x}+e^{-i p_{2} \cdot x} t(-i h \nabla)\right) \frac{1}{z-k_{0}} e^{i p_{2} \cdot x} d z
\end{aligned}
$$

Note that $D_{p_{1}, p_{2}}$ is translation-invariant. That is, $D_{p_{1}, p_{2}}=D_{p_{1}, p_{2}}(-i h \nabla)$ is a multiplication operator in Fourier space, given by

$$
\begin{aligned}
D_{p_{1}, p_{2}}(q)= & \left(t(q)+t\left(q+h p_{1}\right)\right)\left(t(q)+t\left(q+h p_{2}\right)\right) \\
& \times\left[-\beta\left(q^{2}-\mu\right), \beta\left(\left(q+h p_{1}\right)^{2}-\mu\right), \beta\left(\left(q+h p_{2}\right)^{2}-\mu\right)\right]_{f} .
\end{aligned}
$$

Its local trace norm thus equals

$$
\left\|D_{p_{1}, p_{2}}\right\|_{1}=h^{-d} \int_{\mathbb{R}^{d}}\left|D_{p_{1}, p_{2}}(q)\right| \frac{d q}{(2 \pi)^{d}} .
$$

A simple Schwarz inequality and an application of (7.5) show that

$$
\left\|D_{p_{1}, p_{2}}\right\|_{1} \leq C h^{-d} \int_{\mathbb{R}^{d}} \frac{t(q)^{2}}{1+q^{2}} d q,
$$

independently of $p_{1}$ and $p_{2}$. Finally, we can bound $\sum_{p}|\widehat{\psi}(p)| \leq C\|\psi\|_{H^{2}}$ for $d \leq 3$. We have thus shown that

$$
\left\|B_{1}^{a}\right\|_{1} \leq C h^{2-d}\|\psi\|_{H^{2}}^{2} \int_{\mathbb{R}^{d}} \frac{t(q)^{2}}{1+q^{2}} d q .
$$

This concludes the bound on the trace norm of $B_{1}^{a}$. 
The term $B_{1}^{b}$ will be treated very similarly. Recall from (7.31) that $k_{0}-\bar{k}$ is given by a sum of four terms. Let us first look at the two terms linear in $A$. We expand both $\psi$ and $A$ in a Fourier series and conclude that

$$
\left\|B_{1}^{b}\right\|_{1} \leq \frac{h^{3}}{4} \sum_{p_{1}, p_{2}, p_{3} \in(2 \pi \mathbb{Z})^{d}}\left|\widehat{\psi}\left(p_{1}\right)\right|\left|\widehat{\psi}\left(p_{2}\right)\left\|\widehat{A}\left(p_{3}\right) \mid\right\| G_{p_{1}, p_{2}, p_{3}} \|_{1},\right.
$$

where $G_{p_{1}, p_{2}, p_{3}}(-i h \nabla)$ is a multiplication operator in Fourier space, given by

$$
\begin{aligned}
& G_{p_{1}, p_{2}, p_{3}}(q)=\left(t(q)+t\left(q+h p_{1}\right)\right)\left(t\left(q-h p_{3}\right)+t\left(q+h p_{2}-h p_{3}\right)\right)\left(2 q-h p_{3}\right) \\
& \times\left[-\beta\left(q^{2}-\mu\right),-\beta\left(\left(q-h p_{3}\right)^{2}-\mu\right), \beta\left(\left(q+h p_{1}\right)^{2}-\mu\right), \beta\left(\left(q+h\left(p_{2}-p_{3}\right)\right)^{2}-\mu\right)\right]_{f} .
\end{aligned}
$$

To bound its trace norm, we can again use the bounds on the divided differences given in Lemma 6. We shall also need the following additional bound. From the definition (7.2) it follows that if $f$ is Lipschitz continuous, then

$$
\left|\left[a_{1}, a_{2}, a_{3}, a_{4}\right]_{f}\right| \leq \frac{C}{\left|a_{1}-a_{4}\right|}\left(\frac{1}{\left|a_{1}-a_{3}\right|}+\frac{1}{\left|a_{2}-a_{4}\right|}\right) .
$$

If we apply this with $a_{1} \leq a_{2} \leq 0 \leq a_{3} \leq a_{4}$, we obtain the upper bound $C /\left(b_{1} b_{2}\right)$, where $b_{1}$ and $b_{2}$ are, respectively, the largest and second largest among the numbers $\left\{\left|a_{1}\right|,\left|a_{2}\right|,\left|a_{3}\right|,\left|a_{4}\right|\right\}$. In combination with (17.5) one easily obtains the bound

$$
\begin{aligned}
& \left|\left[-\beta\left(q^{2}-\mu\right),-\beta\left(\left(q-h p_{3}\right)^{2}-\mu\right), \beta\left(\left(q+h p_{1}\right)^{2}-\mu\right), \beta\left(\left(q+h\left(p_{2}-p_{3}\right)\right)^{2}-\mu\right)\right]_{f}\right| \\
& \leq \frac{C}{1+q^{2}} \frac{1}{1+\max \left\{\left(q-h p_{3}\right)^{2},\left(q+h p_{1}\right)^{2},\left(q+h p_{2}-h p_{3}\right)^{2}\right\}} .
\end{aligned}
$$

A simple Schwarz inequality then shows that the trace norm of $G_{p_{1}, p_{2}, p_{3}}$ is bounded by $C\left(1+\left|p_{3}\right|\right)$ for some constant $C$ independent of $p_{1}, p_{2}$ and $p_{3}$. Since both $|\widehat{\psi}(p)|$ and $|\widehat{A}(p)|(1+|p|)$ are summable, this shows that this part of $B_{1}^{b}$ is locally trace class. The other part, where the term quadratic in $A$ and the term with $W$ in $k_{0}-\bar{k}$ are taken into account, can be treated in the same way, using our assumption that $|\widehat{W}(p)|$ is summable.

The same method can be used to show that $B_{1}^{c}$ is locally trace class. One simply expands all multiplication operators in Fourier series and then bounds the resulting translation-invariant operator. We omit the details.

We are left with the term $B_{1}^{d}$. We first note that since

$$
f(\beta z)=f(-\beta z)+\beta z
$$

we can replace $f(\beta z)$ by $f(-\beta z)$ in the integrand without changing the integral, since the expression with $\beta z$ in place of $f(\beta z)$ integrates to zero, as can be easily be seen from (7.4), for instance. We can bound the trace norm of the integrand with the aid of Hölder's inequality and (7.33) -(7.35) by a constant times $|z|^{-13 / 6}$ for $\operatorname{Re} z>0$, and $|z|^{1 / 2}$ for $\operatorname{Re} z<0$, respectively. Since we are integrating against a function that decays exponentially for negative Re $z$ and increases linearly for positive Re $z$, the integral is absolutely convergent. This proves that $B_{1}^{d}$ is locally trace class. 


\section{ACKNOWLEDGMENTS}

Part of this work was carried out at the Erwin Schrödinger Institute for Mathematical Physics in Vienna, Austria, and the authors are grateful for the support and hospitality during their visit. The third author would also like to thank the Departamento de Fisica at the Pontificia Universidad Católica de Chile for their hospitality.

\section{REFERENCES}

[1] M. Abramowitz, I.A. Stegun, Handbook of mathematical functions with formulas, graphs, and mathematical tables, Dover (1964). MR0167642 (29:4914)

[2] J. Bardeen, L. Cooper, J. Schrieffer, Theory of Superconductivity, Phys. Rev. 108, 1175-1204 (1957). MR0095694(20:2196)

[3] W.F. Donoghue, Jr., Monotone Matrix Functions and Analytic Continuation, Springer (1974). MR0486556 (58:6279)

[4] S. Fournais, B. Helffer, Spectral Methods in Surface Superconductivity, Birkhäuser (2010). MR2662319 (2011j:35003)

[5] R.L. Frank, C. Hainzl, S. Naboko, R. Seiringer, The critical temperature for the BCS equation at weak coupling, J. Geom. Anal. 17, 559-568 (2007). MR2365659 (2008k:82147)

[6] P.G. de Gennes, Superconductivity of Metals and Alloys, Westview Press (1966).

[7] V.L. Ginzburg, L.D. Landau, On the theory of superconductivity, Zh. Eksp. Teor. Fiz. 20, 1064-1082 (1950).

[8] L.P. Gor'kov, Microscopic derivation of the Ginzburg-Landau equations in the theory of superconductivity, Zh. Eksp. Teor. Fiz. 36, 1918-1923 (1959); English translation Soviet Phys. JETP 9, 1364-1367 (1959).

[9] S. Gustafson, I.M. Sigal, T. Tzaneteas, Statics and dynamics of magnetic vortices and of Nielsen-Olesen (Nambu) strings, J. Math. Phys. 51, 015217 (2010). MR2605850 (2011b:82093)

[10] C. Hainzl, E. Hamza, R. Seiringer, J.P. Solovej, The BCS functional for general pair interactions, Commun. Math. Phys. 281, 349-367 (2008). MR2410898(2009d:82163)

[11] C. Hainzl, M. Lewin, R. Seiringer, A nonlinear theory for relativistic electrons at positive temperature, Rev. Math. Phys. 20, 1283-1307 (2008). MR2466817 (2010g:81308)

[12] C. Hainzl, M. Lewin, É. Séré, Existence of a stable polarized vacuum in the Bogoliubov-DiracFock approximation, Commun. Math. Phys. 257, 515-562 (2005). MR2164942(2006i:81123)

[13] C. Hainzl, R. Seiringer, Critical temperature and energy gap in the BCS equation, Phys. Rev. B 77, 184517 (2008).

[14] C. Hainzl, R. Seiringer, The BCS critical temperature for potentials with negative scattering length. Lett. Math. Phys. 84, 99-107 (2008). MR2415542(2009d:82164)

[15] C. Hainzl, R. Seiringer, Spectral properties of the BCS gap equation of superfluidity, in: Mathematical Results in Quantum Mechanics, proceedings of QMath10, Moeciu, Romania, September 10-15, 2007, World Scientific (2008). MR2466682(2010b:82073)

[16] C. Hainzl, R. Seiringer, Low Density Limit of BCS Theory and Bose-Einstein Condensation of Fermion Pairs, preprint arXiv:1105.1100, Lett. Math. Phys. (in press).

[17] B. Helffer, D. Robert, Calcul fonctionnel par la transformation de Mellin et opérateurs admissibles, J. Funct. Anal. 53, 246-268 (1983). MR724029 (85i:47052)

[18] A.J. Leggett, Diatomic Molecules and Cooper Pairs, in: Modern trends in the theory of condensed matter, A. Pekalski, R. Przystawa, eds., Springer (1980). MR583566 (81h:82002)

[19] A.J. Leggett, Quantum Liquids, Oxford (2006).

[20] E.H. Lieb, M. Loss, Analysis, $2^{\text {nd }}$ ed., Grad. Studies in Math., vol. 14, Amer. Math. Soc. (2001). MR 1817225(2001i:00001)

[21] E.H. Lieb, R. Seiringer, The Stability of Matter in Quantum Mechanics, Cambridge (2010). $\operatorname{MR} 2583992(2011 \mathrm{j}: 81369)$

[22] M. Reed, B. Simon, Methods of Modern Mathematical Physics. IV. Analysis of Operators, Academic Press (1978). MR0493421(58:12429c)

[23] D. Robert, Autour de l'approximation semi-classique, Progress in Mathematics 68, Birkhäuser (1987). MR897108 (89g:81016) 
[24] E. Sandier, S. Serfaty, Vortices in the Magnetic Ginzburg-Landau Model, Birkhäuser (2006). MR.2279839 (2008g:82149)

[25] B. Simon, Trace ideals and their applications, $2^{\text {nd }}$ ed., Mathematical Surveys and Monographs, vol. 120, Amer. Math. Soc. (2005). MR2154153 (2006f:47086)

[26] W. Thirring, Quantum Mathematical Physics, 2 ${ }^{\text {nd }}$ ed., Springer (2002). MR 2133871 (2006b:81368)

Department of Mathematics, Princeton University, Princeton, New Jersey 08544

E-mail address: rlfrank@math.princeton.edu

Mathematisches Institut, Universität Tübingen, Auf Der Morgenstelle 10, 72076 TüBingen, Germany

E-mail address: christian.hainzl@uni-tuebingen.de

Department of Mathematics and Statistics, McGill University, 805 Sherbrooke Street West, Montreal, QC H3A 2K6, Canada

E-mail address: robert.seiringer@mcgill.ca

Department of Mathematics, University of Copenhagen, Universitetsparken 5, DK2100 Copenhagen, Denmark

E-mail address: solovej@math.ku.dk 